Supplementary Information

\title{
Convenient preparation and detailed analysis of a series of NHC-stabilized phosphorus(I) dyes and their derivatives
}

Charles L.B. Macdonald*, Justin F. Binder, Ala'aeddeen Swidan, Jennifer H. Nguyen, Stephanie M. Kosnik, Bobby D. Ellis

Department of Chemistry and Biochemistry, University of Windsor, Windsor, Ontario, Canada N9B 3P4

Metrical Parameters of Phosphamethine Cyanines and Related Species

S2

Crystallographic Data and Structure Refinement Summary

S4

Computational Information

$\mathrm{S} 21$

Cyclic Voltammetry of $\left[\left({ }^{\mathrm{Me}} \mathrm{NHC}{ }^{\mathrm{Me}}\right)_{2} \mathbf{P}\right][\mathrm{OTf}]$

S33

Carbene and $\mathrm{PX}_{3}$ Reaction Results

S34

References

S44 


\section{Metrical Parameters of Phosphamethine Cyanines and Related Species}

Table S1: Selected metrical parameters for structurally characterized acyclic phosphamethine cyanine cations and related species.

\begin{tabular}{|c|c|c|c|c|}
\hline Compound & P-C bonds $(\AA)$ & C-P-C angle (o) & $\begin{array}{l}\text { Carb }_{p}-\mathrm{CPC}_{p} \text { angles } \\
(\underline{\mathrm{o}})^{*}\end{array}$ & Ref. \\
\hline$[\mathrm{A}]\left[\mathrm{ClO}_{4}\right]$ & $1.75(4), 1.75(7)$ & 104.6 & $2.7,4.4$ & 1 \\
\hline$[\mathrm{K}][\mathrm{OTf}]$ & $1.773(3), 1.818(3)$ & $109.2(1)$ & $10.0,11.8$ & 2 \\
\hline$[\mathrm{L}][\mathrm{I}]$ & $1.7866(15)$ & $102.23(10)$ & 10.6 & 3 \\
\hline$[\mathbf{M}]\left[\mathrm{ClO}_{4}\right]$ & $1.77,1.81$ & 100.4 & $24.8,38.8$ & 4 \\
\hline$[\mathrm{N}]\left[\mathrm{ClO}_{4}\right]$ & $1.796(4)[\mathrm{avg}]$ & $103.6(2)$ & $32.0,33.8$ & 5 \\
\hline$\left[\left(\text { iPr } \mathrm{NHC}^{\mathrm{Me}}\right)_{2} \mathrm{P}\right][\mathrm{Cl}]$ & $1.824(2), 1.823(2)$ & $97 \cdot 35(9)$ & $51.7,60.2$ & 6 \\
\hline$\left[\left(\mathrm{MeNHC}^{\mathrm{Me}}\right)_{2} \mathrm{P}\right]\left[(\mathrm{TerN})_{2} \mathrm{P}\right]$ & $1.790(2), 1.801(2)$ & $96.96(7)$ & $40.2,46.3$ & 7 \\
\hline$\left[\left(\mathrm{MeNHC}^{\mathrm{H}}\right)_{2} \mathrm{P}\right][\mathrm{OTf}]$ & $1.796(1), 1.796(1)$ & $98.16(6)$ & $36.3,44.9$ & 8 \\
\hline (two cations in asym. unit) & $1.796(2), 1.800(1)$ & $96.30(6)$ & $43.2,47.6$ & \\
\hline$\left[(\mathrm{MeNHCMe})_{2} \mathrm{P}\right][\mathrm{I}]$ & $1.8036(15), 1.8038(15)$ & $97 \cdot 53(7)$ & $42.8,43.3$ & 8 \\
\hline$\left[(\mathrm{MeNHCMe})_{2} \mathrm{P}\right][\mathrm{Br}]$ & $1.8002(13), 1.8032(13)$ & $98.63(6)$ & $38.8,42.8$ & This work \\
\hline$\left[\left(\mathrm{MeNHC}^{\mathrm{Me}}\right)_{2} \mathrm{P}\right]\left[\mathrm{BPh}_{4}\right]$ & $1.801(2), 1.812(2)$ & $98.30(10)$ & $44.7,59.0$ & This work \\
\hline$\left[\left({ }^{\mathrm{Et}} \mathrm{NHC}{ }^{\mathrm{Me}}\right)_{2} \mathrm{P}\right][\mathrm{Br}]$ & $1.837(6), 1.842(6)$ & $97.2(3)$ & $55 \cdot 5,57.1$ & This work \\
\hline$\left[\left({ }^{\mathrm{iPr}} \mathrm{NHC} \mathrm{Ce}^{\mathrm{Me}}\right)_{2} \mathrm{P}\right][\mathrm{Br}]$ & $1.8195(16), 1.8225(16)$ & $100.44(7)$ & $54.7,60.9$ & This work \\
\hline$\left[\left(\mathrm{MeNHC}^{\mathrm{B}}\right)_{2} \mathrm{P}\right][\mathrm{Br}]$ & $1.787(3), 1.795(3)$ & $99.27(14)$ & $35.4,42.8$ & This work \\
\hline$\left[\left(\mathrm{MeNHC}^{\mathrm{B}}\right)_{2} \mathrm{P}\right][\mathrm{I}]$ & $1.7875(19), 1.793(2)$ & $99.25(8)$ & $33.1,42.1$ & This work \\
\hline (two cations in asym. unit) & $1.775(2), 1.804(2)$ & 98.49(9) & $30.5,48.4$ & \\
\hline$\left[\left(\mathrm{MeNHC}^{\mathrm{B}}\right)_{2} \mathrm{P}\right][\mathrm{OTf}]$ & $1.783(3), 1.787(3)$ & $99 \cdot 36(12)$ & $35 \cdot 7,35.8$ & This work \\
\hline$\left[\left(\mathrm{Et}^{\mathrm{N} H C^{\mathrm{B}}}\right)_{2} \mathrm{P}\right][\mathrm{Br}]$ & $1.798(2), 1.799(2)$ & $98.78(10)$ & $37.9,51.9$ & This work \\
\hline$\left[\left({ }^{\left(P^{2}\right.} \mathrm{NHC}^{\mathrm{B}}\right)_{2} \mathrm{P}\right][\mathrm{Br}]$ & $1.8002(13), 1.8032(13)$ & $98.63(6)$ & $36.6,59.8$ & This work \\
\hline $\mathbf{O}$ & $1.784(5), 1.778(4)$ & $98.7(2)$ & $3.772,6.770$ & 9 \\
\hline $\mathbf{P}$ & $1.786(2), 1.782(2)$ & $106.60(7)$ & $15 \cdot 58,16.68$ & 10 \\
\hline $\mathbf{Q}$ & $1.779(2)$ & $104.2(1)$ & $3.604,4.870$ & 11 \\
\hline $\mathbf{R}$ & $1.783,1.784$ & 106.91 & $6.227,15.177$ & 12 \\
\hline $\mathbf{S}$ & $1.745(3), 1.794(3)$ & $103.4(1)$ & $0.00,0.00$ & 13 \\
\hline $\left.3{ }^{[\mathrm{Me}} \mathrm{T}\right][\mathrm{Br}]$ & $1.778(3)$ to $1.795(2)$ & $91.43(10)$ to $93.13(11)$ & $15 \cdot 77$ to $25 \cdot 38$ & 14 \\
\hline$\left[{ }^{\mathrm{Me}} \mathrm{T}\right][\mathrm{OTf}]$ & $1.7810(14), 1.7813(15)$ & $92.07(6)$ & $18.83,19.10$ & 14 \\
\hline$\left[{ }^{B n} \mathbf{T}\right][\mathrm{Br}]$ & $1.793(2)$ & $91.87(11)$ & $25.02,25.47$ & 14 \\
\hline$\left[{ }^{\mathrm{BnT}}\right]\left[\mathrm{BPh}_{4}\right](\cdot \mathrm{THF})$ & $1.7797(16), 1.7811(16)$ & $92.51(7)$ & $18.74,19.51$ & 14 \\
\hline$\left[{ }^{\mathrm{BnT}}\right]\left[\mathrm{BPh}_{4}\right](\cdot \mathrm{DCM})$ & $1.782(2), 1.790(2)$ & $92.08(9)$ & $21.67,22.36$ & 14 \\
\hline
\end{tabular}

*These values are the angles $\left(^{\circ}\right)$ between the $C$-P-C plane and the respective $N$ - $C$-X planes of the two heterocycles, where $X$ is $N$, $S$, or $C$, and they are determined using the program Mercury. 
Figure S1. Depictions of structurally characterized acyclic phosphamethine cyanine cations and related species. The systems investigated in this work are boxed.<smiles>CCn1c(=Pc2sc3ccccc3[n+]2CC)sc2ccccc21</smiles>

A

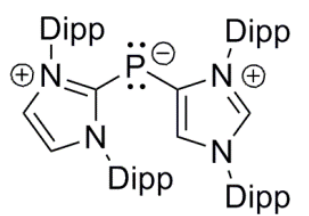

K

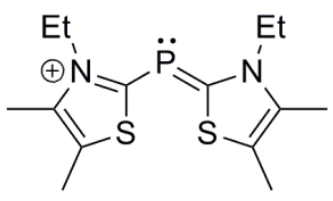

L<smiles>CCn1c(=Pc2ccc3ccccc3[n+]2CC)ccc2ccccc21</smiles>

M<smiles>CN(C)C(=[N+](C)C)[PH](=O)C(N(C)C)N(C)C</smiles>

N

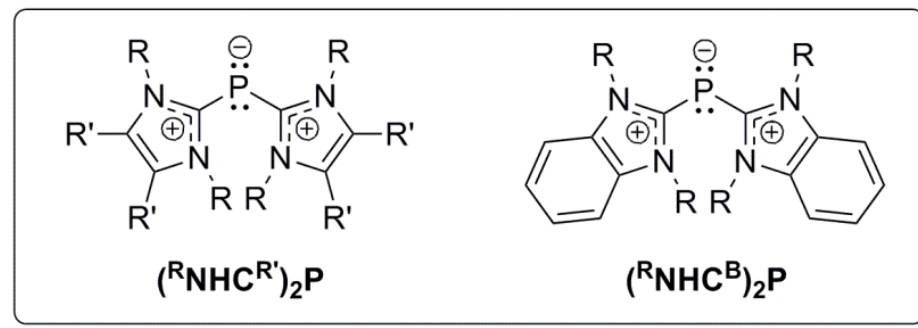<smiles>c1ccc2sc(/P=c3\[nH]c4ccccc4s3)nc2c1</smiles>

O

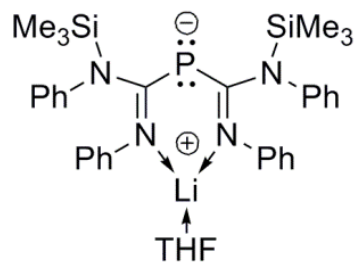

$\mathbf{R}$

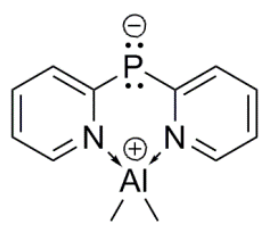

$\mathbf{P}$<smiles></smiles>

$\mathrm{R}_{\mathbf{T}}$ 


\section{Crystallographic Data and Structure Refinement Summary}

Figure S2. Thermal ellipsoid plot of $\left[\left({ }^{\mathrm{Me}} \mathrm{NHC} \mathrm{Me}_{2} \mathrm{P}\right][\mathrm{Br}] \cdot \mathrm{MeCN}\right.$. Ellipsoids are drawn using the $30 \%$ probability surface.

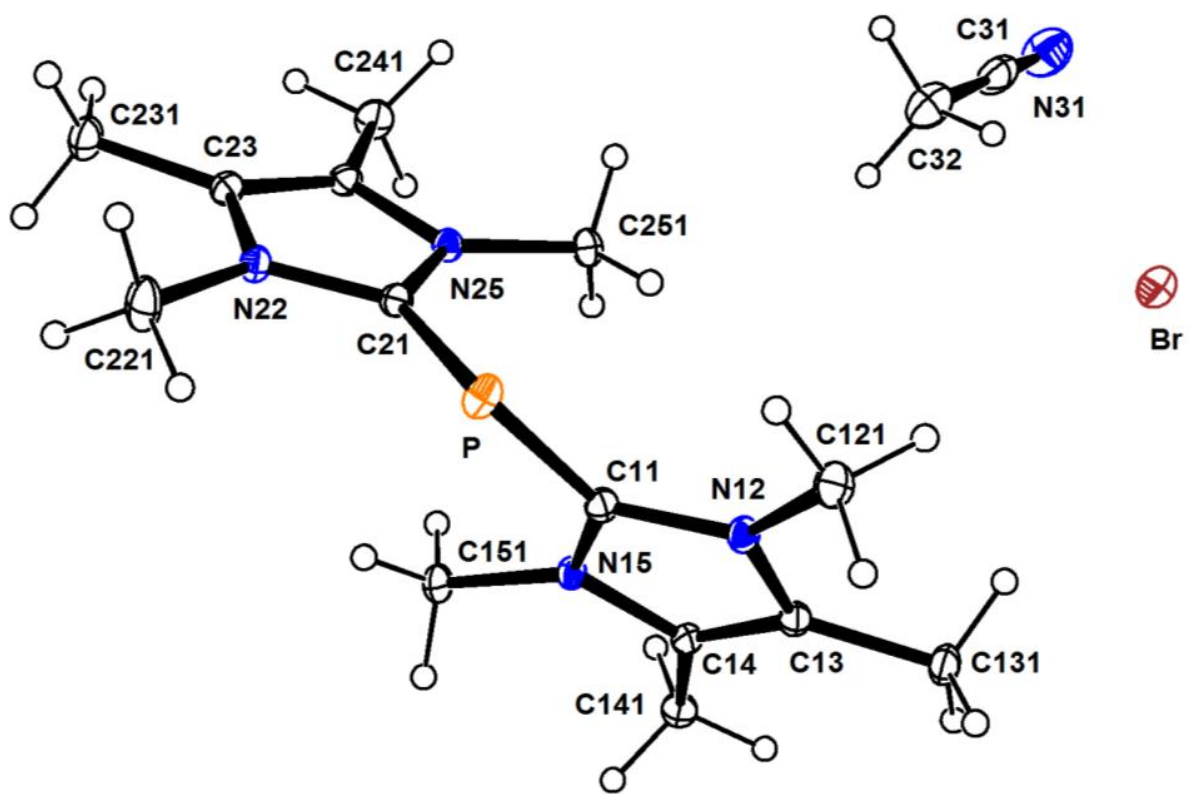

Table S2. Crystal data and structure refinement for $\left[\left({ }^{\mathrm{Me}} \mathrm{NHC}{ }^{\mathrm{Me}}\right)_{2} \mathrm{P}\right][\mathrm{Br}] \bullet \mathrm{MeCN}$.

Identification code

Empirical formula

Formula weight

Temperature

Wavelength

Crystal system

Space group

Unit cell dimensions

shelx

$\mathrm{C}_{16} \mathrm{H}_{27} \mathrm{BrN}_{5} \mathrm{P}$

400.30

173(2) K

$0.71073 \AA$

Monoclinic

$\mathrm{P} 21 / \mathrm{c}$

$\mathrm{a}=6.9633(3) \AA$

$\mathrm{a}=90^{\circ}$.

$\mathrm{b}=12.1035(5) \AA$

$\mathrm{c}=22.8650(8) \AA$

Volume

1907.71(13) $\AA^{3}$

$\mathrm{b}=98.1280(10)^{\circ}$.

4

Density (calculated)

$1.394 \mathrm{Mg} / \mathrm{m}^{3}$

Absorption coefficient

$2.245 \mathrm{~mm}^{-1}$

$\mathrm{F}(000)$

832

Crystal size

Theta range for data collection

$0.192 \times 0.18 \times 0.17 \mathrm{~mm}^{3}$

2.955 to $30.558^{\circ}$.

Index ranges

Reflections collected

$-9<=\mathrm{h}<=9,-17<=\mathrm{k}<=17,-32<=1<=28$

Independent reflections

Completeness to theta $=25.242^{\circ}$

56643

$5825[\mathrm{R}$ (int) $=0.0317]$

$99.9 \%$

Absorption correction

Max. and min. transmission

Semi-empirical from equivalents

0.683 and 0.623

Refinement method

Data / restraints / parameters

Full-matrix least-squares on $\mathrm{F}^{2}$

5825 / 0 / 217

Goodness-of-fit on $\mathrm{F}^{2}$

1.039

Final R indices [I $>2 \operatorname{sigma(I)]~}$

$\mathrm{R}$ indices (all data)

Extinction coefficient

$\mathrm{R} 1=0.0242, \mathrm{wR} 2=0.0579$

$\mathrm{R} 1=0.0342, \mathrm{wR} 2=0.0628$

$\mathrm{n} / \mathrm{a}$

Largest diff. peak and hole

0.373 and -0.268 e. $\AA^{-3}$ 
Figure S3. Thermal ellipsoid plot of $\left[\left({ }^{\mathrm{Me}} \mathrm{NHC}^{\mathrm{Me}}\right)_{2} \mathrm{P}\right]\left[\mathrm{BPh}_{4}\right]$. Ellipsoids are drawn using the $30 \%$ probability surface.

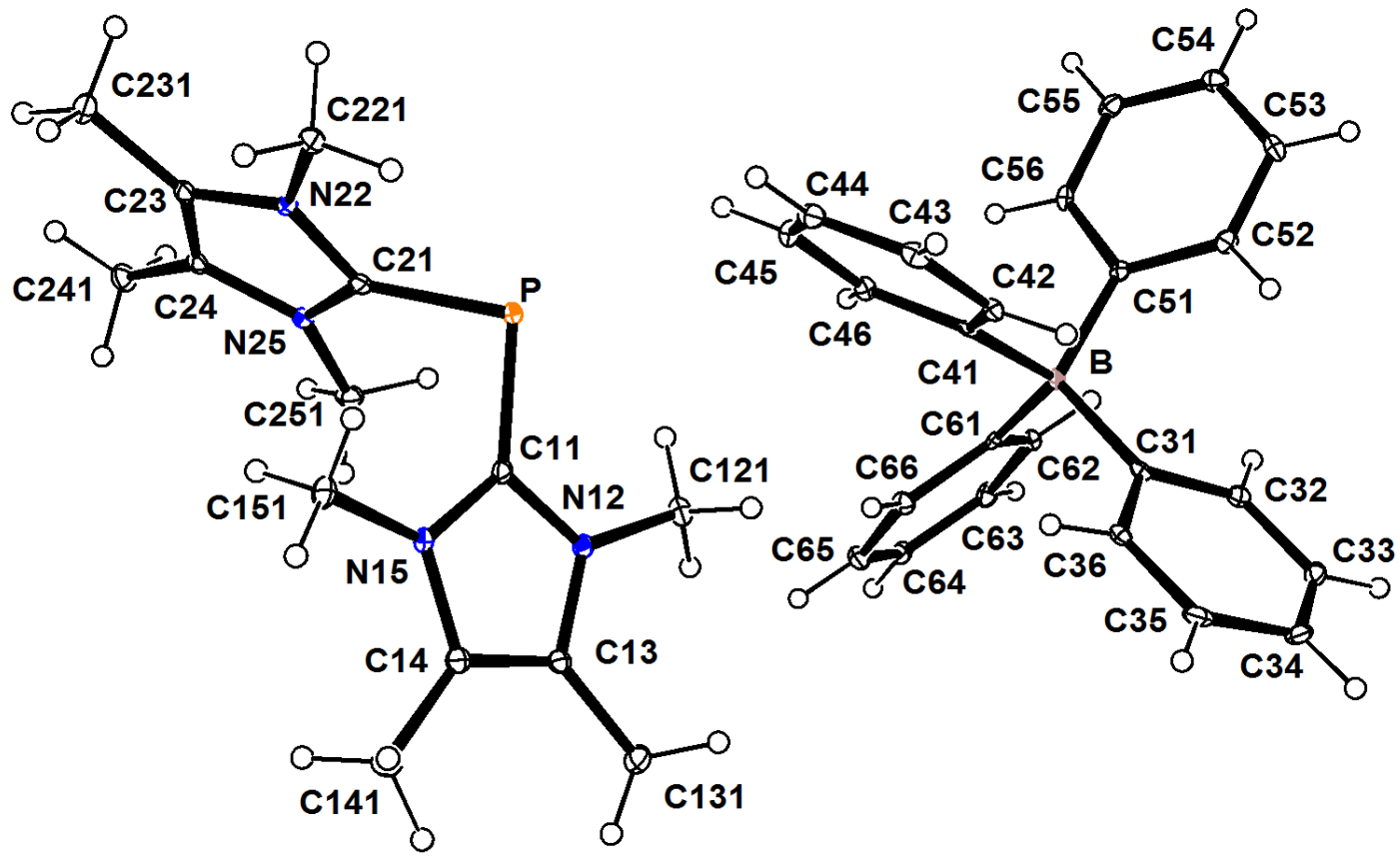

Table S3. Crystal data and structure refinement for $\left[\left({ }^{\mathrm{Me}} \mathrm{NHC}^{\mathrm{Me}}\right)_{2} \mathrm{P}\right][\mathrm{BPh} 4]$.

Identification code

shelx

Empirical formula

$\mathrm{C}_{38} \mathrm{H}_{44} \mathrm{BN}_{4} \mathrm{P}$

Formula weight

Temperature

Wavelength

Crystal system

Space group

Unit cell dimensions

598.55

100(2) K

$0.71073 \AA$

Monoclinic

P 21/n

$\mathrm{a}=9.6887(8) \AA$

$\mathrm{b}=29.759(3) \AA$

$\alpha=90^{\circ}$.

$\mathrm{c}=11.3473(9) \AA$

$\beta=92.616(2)^{\circ}$.

3268.3(5) $\AA^{3}$

Z

4

Density (calculated)

$1.216 \mathrm{Mg} / \mathrm{m}^{3}$

Absorption coefficient

$0.117 \mathrm{~mm}^{-1}$

$\mathrm{F}(000)$

1280

Crystal size

Theta range for data collection

$0.500 \times 0.213 \times 0.193 \mathrm{~mm}^{3}$

2.911 to $27.166^{\circ}$.

Index ranges

Reflections collected

Independent reflections

Completeness to theta $=25.242^{\circ}$

Absorption correction

Max. and min. transmission

Refinement method

Data / restraints / parameters

$-12<=\mathrm{h}<=12,-38<=\mathrm{k}<=38,-14<=\mathrm{l}<=14$

62403

$7237[\mathrm{R}(\mathrm{int})=0.0740]$

$99.8 \%$

Semi-empirical from equivalents

0.978 and 0.897

Full-matrix least-squares on $\mathrm{F}^{2}$

7237 / 0 / 405

Goodness-of-fit on $\mathrm{F}^{2}$

1.155

Final $R$ indices [I $>2 \operatorname{sigma}(\mathrm{I})]$

$\mathrm{R}$ indices (all data)

Extinction coefficient

$\mathrm{R} 1=0.0684, \mathrm{wR} 2=0.1201$

$\mathrm{R} 1=0.0973, \mathrm{wR} 2=0.1298$

$\mathrm{n} / \mathrm{a}$

Largest diff. peak and hole

0.363 and -0.362 e. $\AA^{-3}$ 
Figure S4. Thermal ellipsoid plot of $\left[\left({ }^{\mathrm{Et}} \mathrm{NHC}^{\mathrm{Me}}\right)_{2} \mathrm{P}\right][\mathrm{Br}] \bullet 0.33 \mathrm{MeCN}$. Ellipsoids are drawn using the $30 \%$ probability surface.

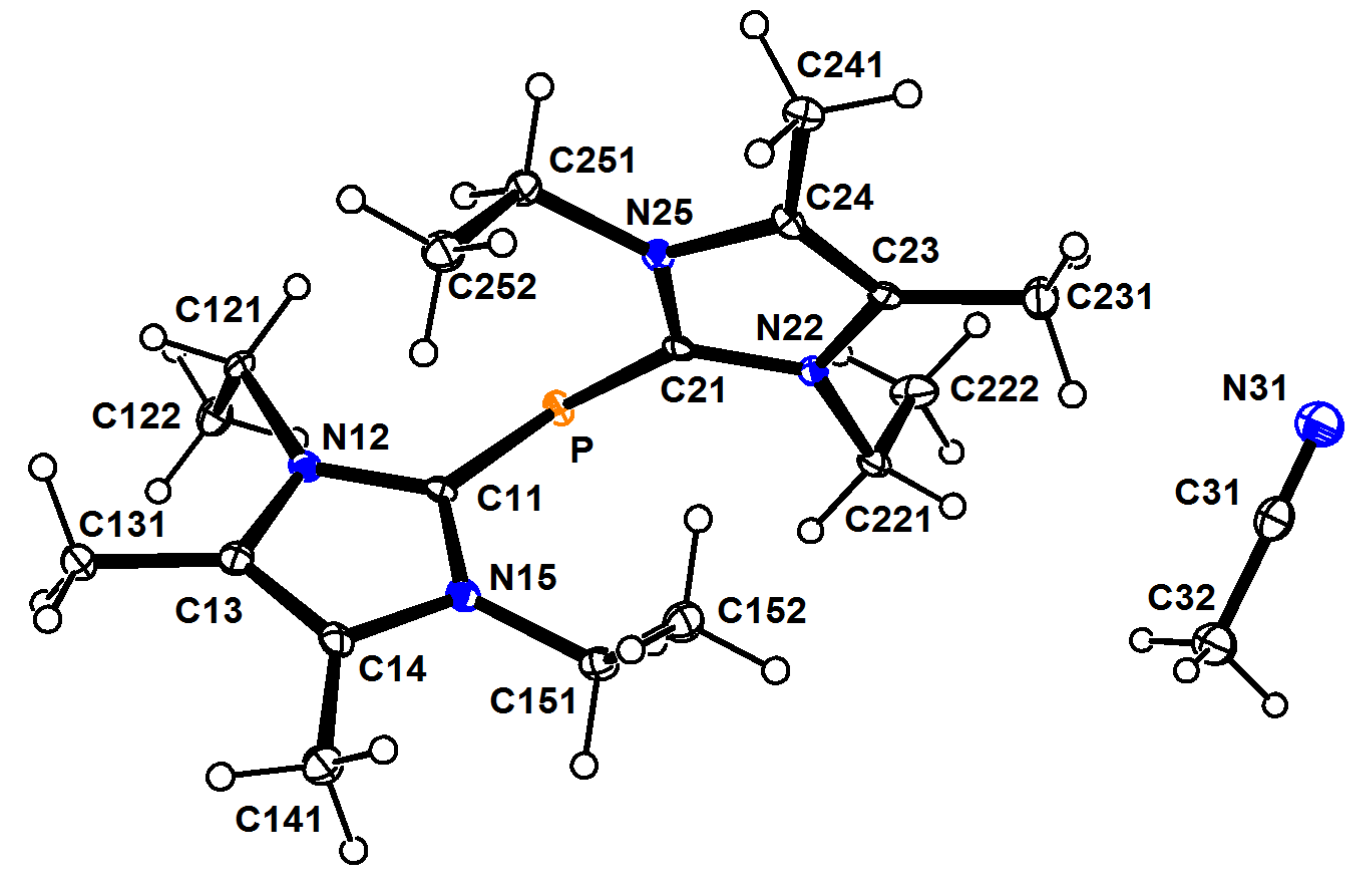

Table S4. Crystal data and structure refinement for $\left[\left({ }^{\mathrm{Et}} \mathrm{NHC}{ }^{\mathrm{Me}}\right)_{2} \mathrm{P}\right][\mathrm{Br}] \bullet 0.33 \mathrm{MeCN}$.

Identification code

Empirical formula

Formula weight

Temperature

Wavelength

Crystal system

Space group

Unit cell dimensions

Volume

Z

Density (calculated)

Absorption coefficient

$\mathrm{F}(000)$

Crystal size

Theta range for data collection

Index ranges

Reflections collected

Independent reflections

Completeness to theta $=25.242^{\circ}$

Absorption correction

Max. and min. transmission

Refinement method

Data / restraints / parameters

Goodness-of-fit on $\mathrm{F}^{2}$

Final $\mathrm{R}$ indices [I $>2 \operatorname{sigma}(\mathrm{I})]$

$\mathrm{R}$ indices (all data)

Absolute structure parameter

Extinction coefficient

Largest diff. peak and hole

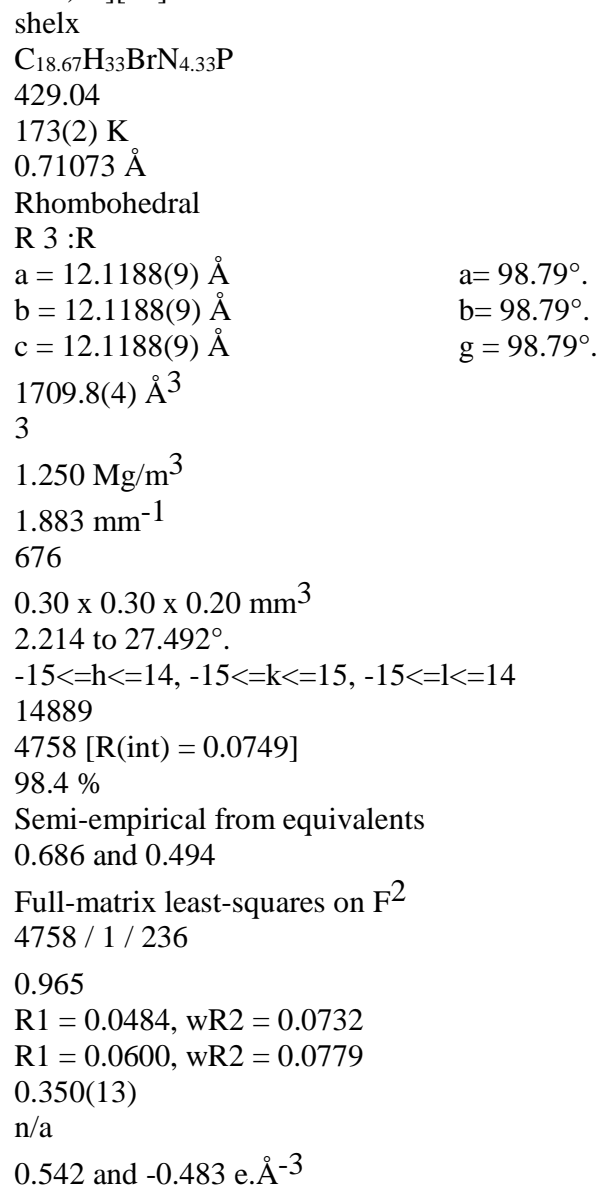


Figure S5. Thermal ellipsoid plot of $\left[\left({ }^{\mathrm{iPr}} \mathrm{NHC}^{\mathrm{Me}}\right)_{2} \mathrm{P}\right][\mathrm{Br}]$. Ellipsoids are drawn using the $30 \%$ probability surface.

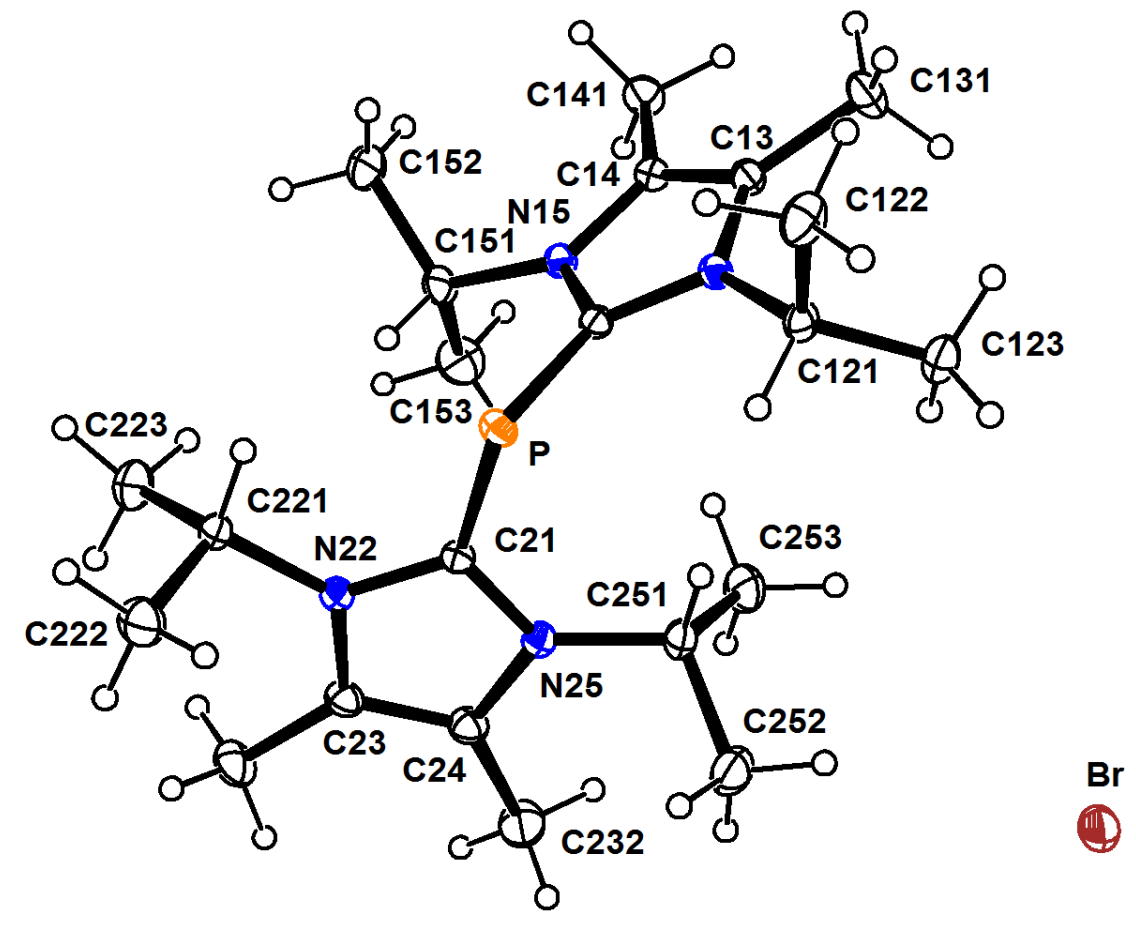

Table S5. Crystal data and structure refinement for $\left[\left({ }^{\mathrm{Pr}} \mathrm{NHC}{ }^{\mathrm{Me}}\right)_{2} \mathrm{P}\right][\mathrm{Br}]$.

Identification code

Empirical formula

Formula weight

Temperature

Wavelength

Crystal system

Space group

Unit cell dimensions

Volume

$\mathrm{Z}$

Density (calculated)

Absorption coefficient

$\mathrm{F}(000)$

Crystal size

Theta range for data collection

Index ranges

Reflections collected

Independent reflections

Completeness to theta $=67.679^{\circ}$

Absorption correction

Max. and min. transmission

Refinement method

Data / restraints / parameters

Goodness-of-fit on $\mathrm{F}^{2}$

Final R indices [I $>2 \operatorname{sigma}(\mathrm{I})]$

$\mathrm{R}$ indices (all data)

Extinction coefficient

Largest diff. peak and hole

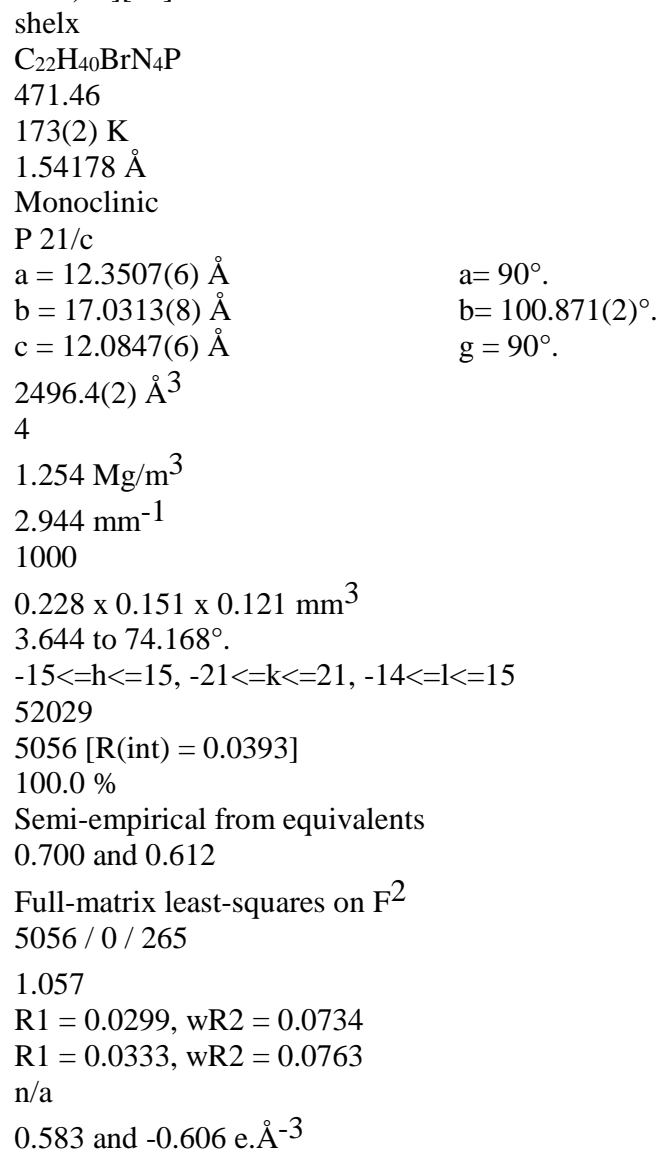


Figure S6. Thermal ellipsoid plot of $\left[\left({ }^{\mathrm{Me}} \mathrm{NHC}^{\mathrm{B}}\right)_{2} \mathrm{P}\right][\mathrm{Br}]$. Ellipsoids are drawn using the $30 \%$ probability surface.

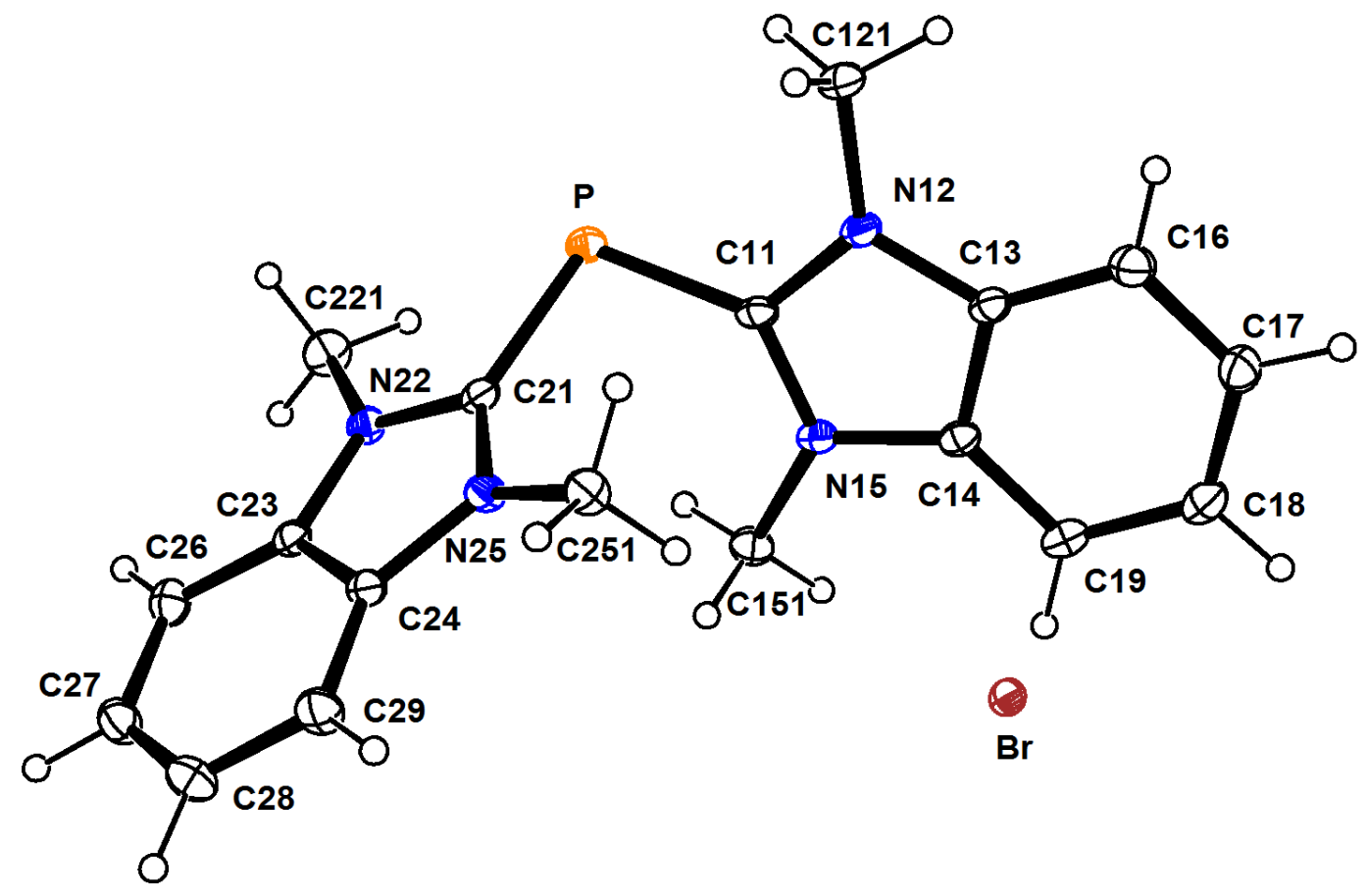

Table S6. Crystal data and structure refinement for $\left[\left({ }^{\mathrm{Me} N H C}{ }_{2}^{\mathrm{B}}\right)_{2} \mathrm{P}\right][\mathrm{Br}]$.

Identification code

shelx

Empirical formula

$\mathrm{C}_{18} \mathrm{H}_{20} \mathrm{BrN}_{4} \mathrm{P}$

Formula weight

Temperature

Wavelength

403.26

Crystal system

Space group

173(2) K

$0.71073 \AA$

Tetragonal

Unit cell dimensions

I $41 \mathrm{c} \mathrm{d}$

$\mathrm{a}=20.0956(7) \AA$

$\mathrm{b}=20.0956(7) \AA$

$\mathrm{a}=90^{\circ}$.

$\mathrm{c}=18.3456(7) \AA$

$\mathrm{b}=90^{\circ}$.

7408.6(6) $\AA^{3}$

Volume

Z

16

Density (calculated)

$1.446 \mathrm{Mg} / \mathrm{m}^{3}$

Absorption coefficient

$2.313 \mathrm{~mm}^{-1}$

$\mathrm{F}(000)$

3296

Crystal size

Theta range for data collection

Index ranges

Reflections collected

Independent reflections

Completeness to theta $=25.242^{\circ}$

Absorption correction

Refinement method

Data / restraints / parameters

Goodness-of-fit on $\mathrm{F}^{2}$

Final R indices [I $>2$ sigma(I)]

$\mathrm{R}$ indices (all data)

Absolute structure parameter

Extinction coefficient

$0.251 \times 0.154 \times 0.141 \mathrm{~mm}^{3}$

3.007 to $27.156^{\circ}$.

$-23<=\mathrm{h}<=25,-25<=\mathrm{k}<=25,-23<=1<=23$

49192

4097 [R(int) $=0.0687]$

$99.8 \%$

None

Full-matrix least-squares on $\mathrm{F}^{2}$

4097 / 1 / 221

1.059

$\mathrm{R} 1=0.0258, \mathrm{wR} 2=0.0494$

$\mathrm{R} 1=0.0374, \mathrm{wR} 2=0.0535$

$-0.003(4)$

Largest diff. peak and hole

$\mathrm{n} / \mathrm{a}$

0.252 and -0.299 e. $\AA^{-3}$ 
Figure S7. Thermal ellipsoid plot of $\left[\left({ }^{\mathrm{Me}} \mathrm{NHC}^{\mathrm{B}}\right)_{2} \mathrm{P}\right][\mathrm{I}]$. Ellipsoids are drawn using the $30 \%$ probability surface.

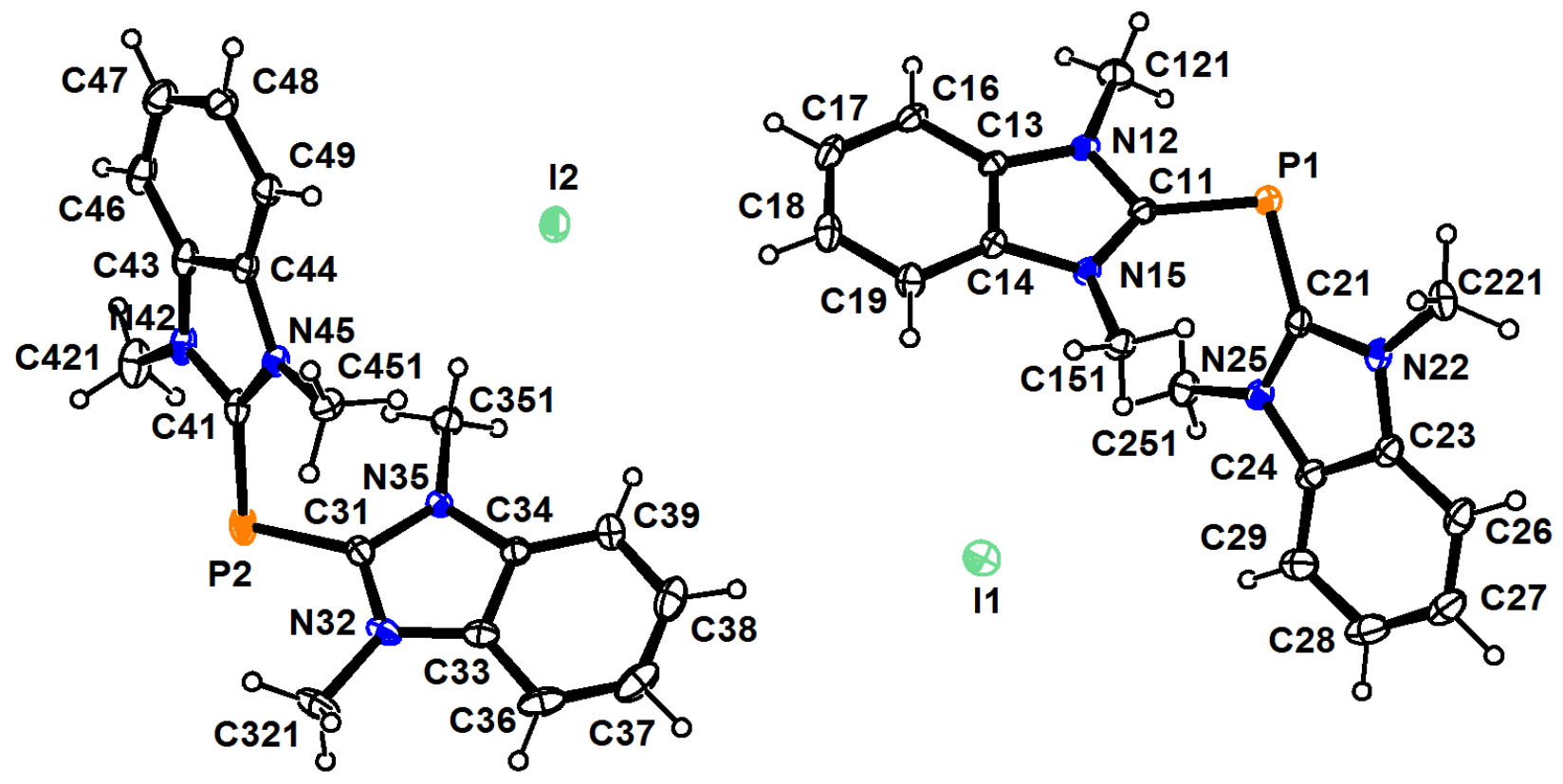

Table S7. Crystal data and structure refinement for $\left[\left({ }^{\mathrm{Me}} \mathrm{NHC}{ }^{\mathrm{B}}\right)_{2} \mathrm{P}\right][\mathrm{I}]$.

Identification code

Empirical formula

Formula weight

Temperature

Wavelength

Crystal system

Space group

Unit cell dimensions

Volume

Z

Density (calculated)

Absorption coefficient $\mathrm{F}(000)$

Crystal size

Theta range for data collection

Index ranges

Reflections collected

Independent reflections

Completeness to theta $=25.242^{\circ}$

Absorption correction

Max. and min. transmission

Refinement method

Data / restraints / parameters

Goodness-of-fit on $\mathrm{F}^{2}$

Final R indices [I $>2 \operatorname{sigma}(\mathrm{I})]$

$\mathrm{R}$ indices (all data)

Extinction coefficient

Largest diff. peak and hole shelx

$\mathrm{C}_{18} \mathrm{H}_{20} \mathrm{IN}_{4} \mathrm{P}$

450.25

173(2) K

$0.71073 \AA$

Monoclinic

P 21/c

$\mathrm{a}=16.6369(6) \AA$

$\mathrm{b}=14.6559(6) \AA$

$\mathrm{c}=16.2198(6) \AA$

3792.8(3) $\AA^{3}$

8

$1.577 \mathrm{Mg} / \mathrm{m}^{3}$

$1.780 \mathrm{~mm}^{-1}$

1792

$0.226 \times 0.162 \times 0.140 \mathrm{~mm}^{3}$

2.780 to $27.500^{\circ}$.

$-21<=\mathrm{h}<=21,-19<=\mathrm{k}<=19,-20<=\mathrm{l}<=21$

90864

$8699[\mathrm{R}($ int $)=0.0245]$

$99.9 \%$

Semi-empirical from equivalents

0.779 and 0.735

Full-matrix least-squares on $\mathrm{F}^{2}$

8699 / 0 / 441

1.048

$\mathrm{R} 1=0.0232, \mathrm{wR} 2=0.0547$

$\mathrm{R} 1=0.0279, \mathrm{wR} 2=0.0579$

$\mathrm{n} / \mathrm{a}$

0.615 and -1.138 e. $\AA^{-3}$

$$
\begin{aligned}
& \mathrm{a}=90^{\circ} . \\
& \mathrm{b}=106.4580(10)^{\circ} . \\
& \mathrm{g}=90^{\circ} .
\end{aligned}
$$


Figure S8. Thermal ellipsoid plot of $\left[\left({ }^{\mathrm{Et}} \mathrm{NHC}^{\mathrm{B}}\right)_{2} \mathrm{P}\right][\mathrm{I}] \cdot 0.36 \mathrm{MeCN}$. Ellipsoids are drawn using the $30 \%$ probability surface.
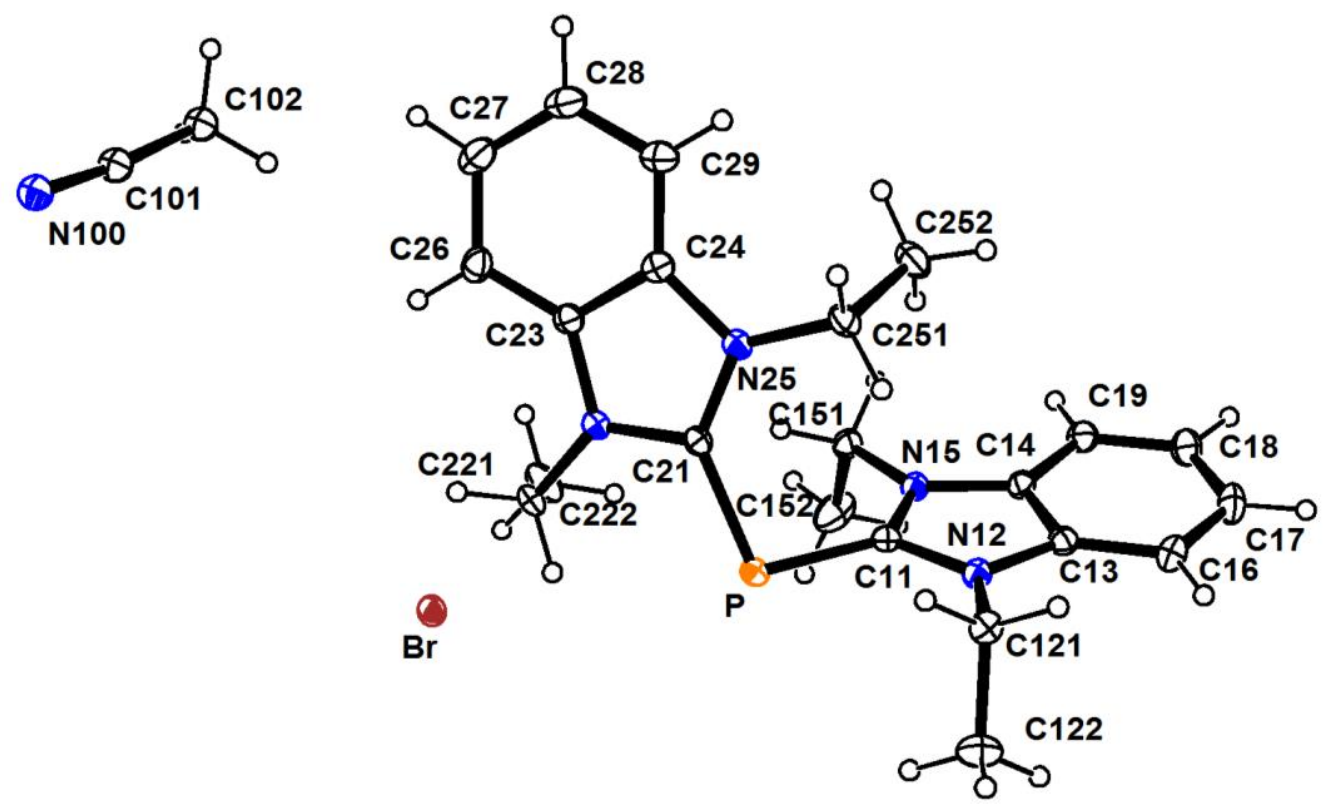

The disorder in the MeCN molecule was modeled over 2 sites with a 50:50 relative occupancy. The overall occupancy was refined to 0.36 per cation (or anion).

Table S8. Crystal data and structure refinement for $\left[\left({ }^{\mathrm{Et}} \mathrm{NHC}{ }^{\mathrm{B}}\right)_{2} \mathrm{P}\right][\mathrm{I}] \cdot 0.36 \mathrm{MeCN}$.

Identification code

Empirical formula

Formula weight

Temperature

Wavelength

Crystal system

Space group

Unit cell dimensions

Volume

Z

Density (calculated)

Absorption coefficient

$\mathrm{F}(000)$

Crystal size

Theta range for data collection

Index ranges

Reflections collected

Independent reflections

Completeness to theta $=25.242^{\circ}$

Absorption correction

Max. and min. transmission

Refinement method

Data / restraints / parameters

Goodness-of-fit on $\mathrm{F}^{2}$

Final R indices [I>2sigma(I)]

$\mathrm{R}$ indices (all data)

Extinction coefficient

Largest diff. peak and hole

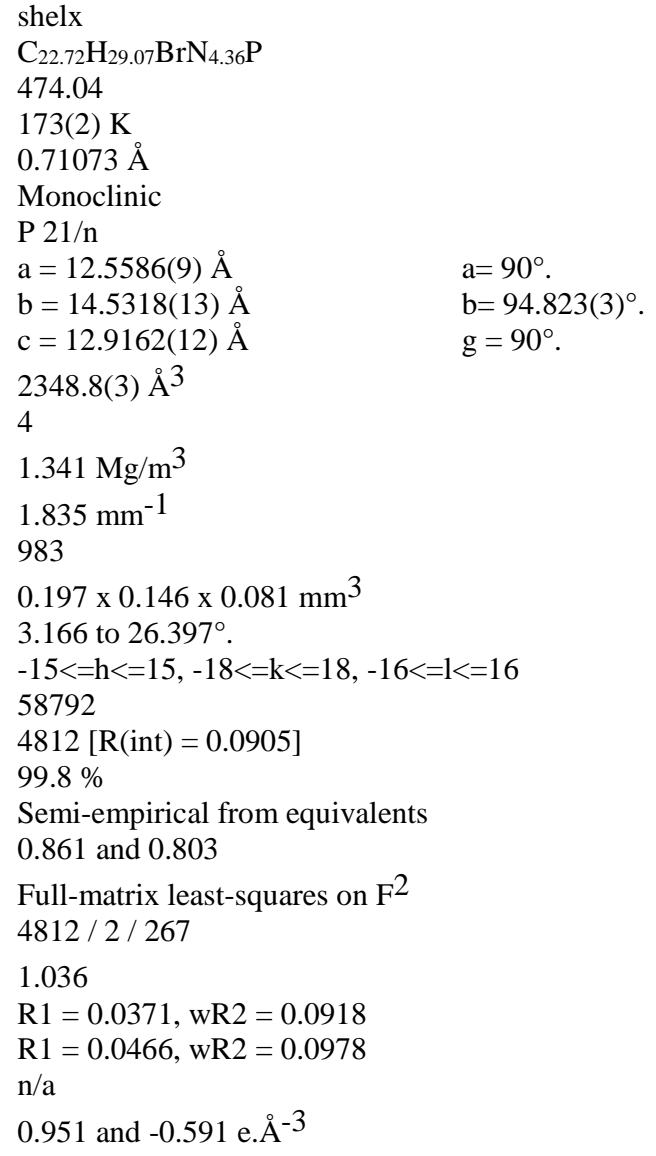


Figure S9. Thermal ellipsoid plot of $\left[\left({ }^{i P r} \mathrm{NHC}^{\mathrm{B}}\right)_{2} \mathrm{P}\right][\mathrm{I}] \bullet \mathrm{MeCN}$. Ellipsoids are drawn using the $30 \%$ probability surface.

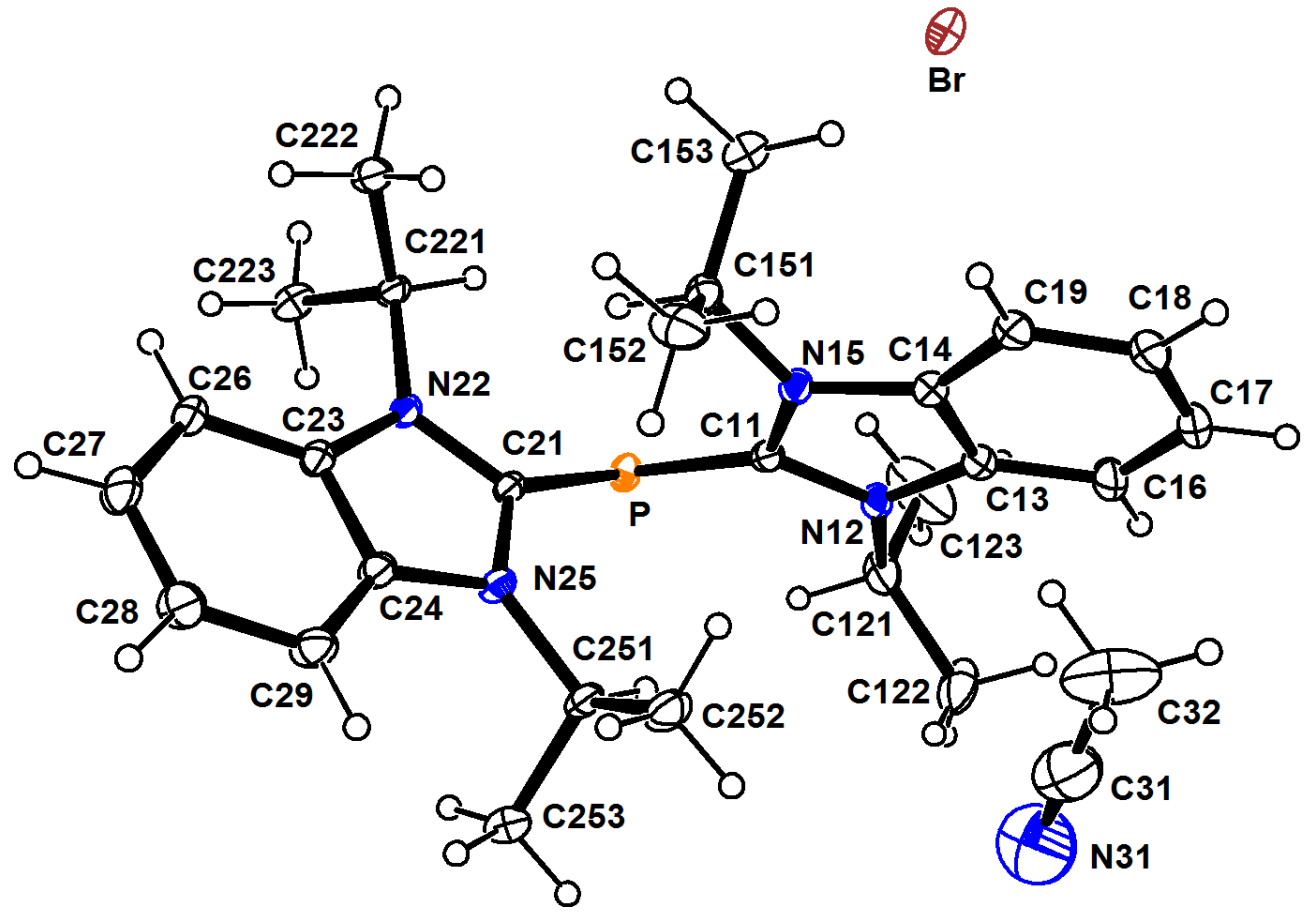

Table S9. Crystal data and structure refinement for $\left[\left({ }^{\left(\mathrm{Pr}^{2}\right.} \mathrm{NHC}{ }^{\mathrm{B}}\right)_{2} \mathrm{P}\right][\mathrm{I}] \cdot \mathrm{MeCN}$.

Identification code

Empirical formula

Formula weight

Temperature

Wavelength

Crystal system

Space group

Unit cell dimensions

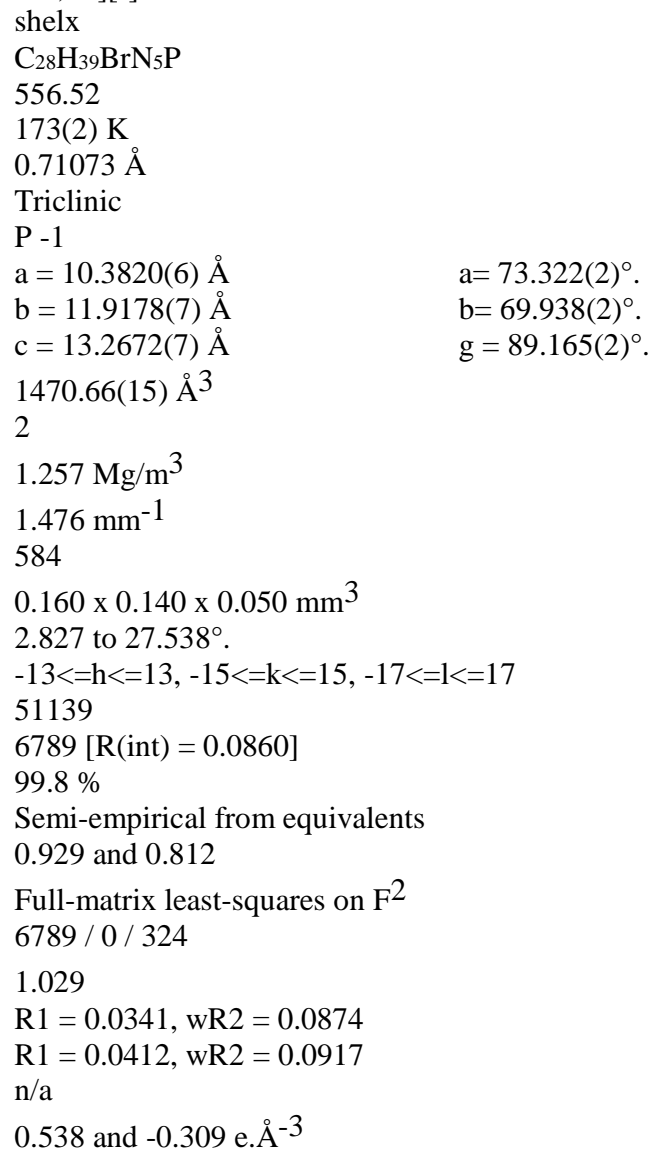


Figure S10. Thermal ellipsoid plot of $\left[\left({ }^{\mathrm{Me}} \mathrm{NHC}^{\mathrm{B}}\right)_{2} \mathrm{P}\right][\mathrm{OTf}]$. Ellipsoids are drawn using the $30 \%$ probability surface.
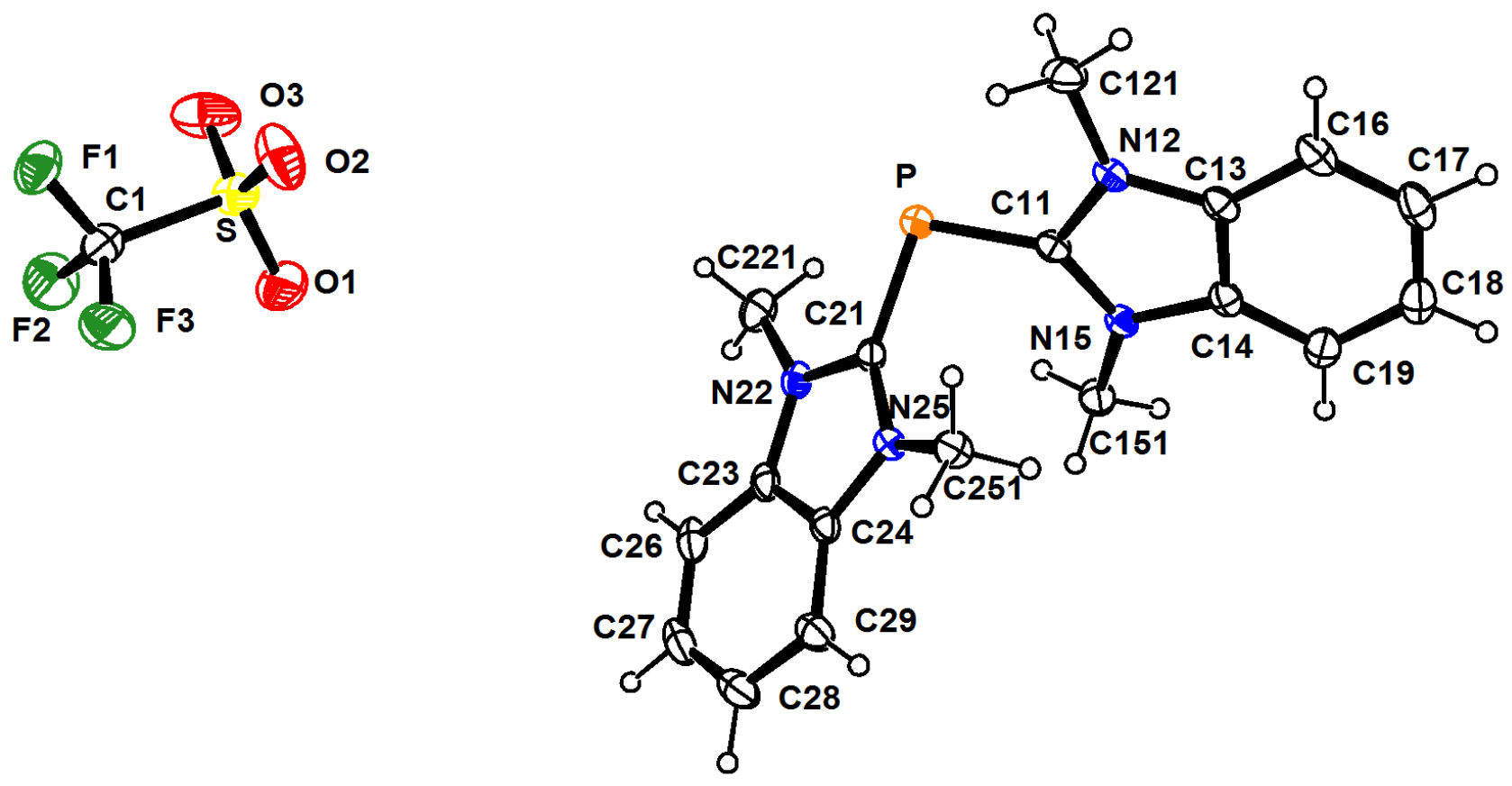

Table S10. Crystal data and structure refinement for $\left[\left({ }^{\mathrm{Me}} \mathrm{NHC}{ }^{\mathrm{B}}\right)_{2} \mathrm{P}\right][\mathrm{OTf}]$.

Identification code

Empirical formula

Formula weight

Temperature

Wavelength

Crystal system

Space group

Unit cell dimensions

Volume

\section{Z}

Density (calculated)

Absorption coefficient

$\mathrm{F}(000)$

Crystal size

Theta range for data collection

Index ranges

Reflections collected

Independent reflections

Completeness to theta $=67.679^{\circ}$

Absorption correction

Max. and min. transmission

Refinement method

Data / restraints / parameters

Goodness-of-fit on $\mathrm{F}^{2}$

Final R indices [I>2sigma(I)]

$\mathrm{R}$ indices (all data)

Extinction coefficient

Largest diff. peak and hole shelx

$\mathrm{C}_{19} \mathrm{H}_{20} \mathrm{~F}_{3} \mathrm{~N}_{4} \mathrm{O}_{3} \mathrm{PS}$

472.42

$173(2) \mathrm{K}$

$1.54178 \AA$

Orthorhombic

$\mathrm{P} \mathrm{b} \mathrm{c} \mathrm{a}$

$\mathrm{a}=15.5193(3) \AA$

$\mathrm{b}=15.7336(3) \AA$

$\mathrm{c}=17.1506(3) \AA$

4187.74(14) $\AA^{3}$

8

$1.499 \mathrm{Mg} / \mathrm{m}^{3}$

$2.611 \mathrm{~mm}^{-1}$

1952

$0.15 \times 0.117 \times 0.085 \mathrm{~mm}^{3}$

4.761 to $74.562^{\circ}$.

$-19<=\mathrm{h}<=19,-19<=\mathrm{k}<=18,-21<=\mathrm{l}<=20$

46106

$4278[\mathrm{R}(\mathrm{int})=0.0874]$

$100.0 \%$

Semi-empirical from equivalents

0.801 and 0.668

Full-matrix least-squares on $\mathrm{F}^{2}$

4278 / 0 / 284

1.026

$\mathrm{R} 1=0.0509, \mathrm{wR} 2=0.1273$

$\mathrm{R} 1=0.0714, \mathrm{wR} 2=0.1418$

$\mathrm{n} / \mathrm{a}$

1.434 and -0.453 e. $\AA^{-3}$ 
Figure S11. Thermal ellipsoid plot of $\left[\left({ }^{\mathrm{Me}} \mathrm{NHC}^{\mathrm{Me}}\right)_{2} \mathrm{PS}_{2}\right][\mathrm{Br}]$. Ellipsoids are drawn using the $30 \%$ probability surface.

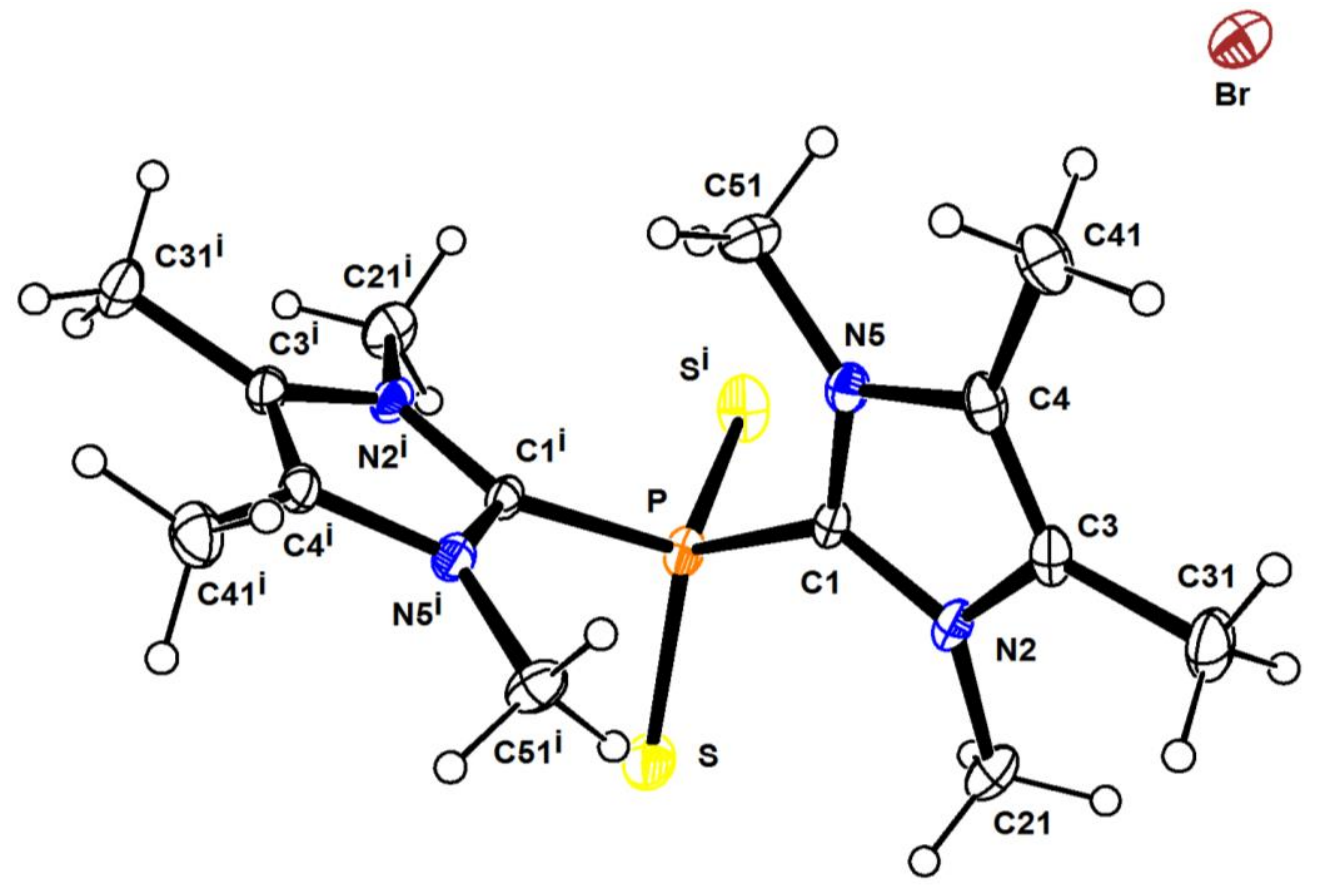

Table S11. Crystal data and structure refinement for $\left[\left({ }^{\mathrm{Me}} \mathrm{NHC} \mathrm{Me}_{2} \mathrm{PS}_{2}\right][\mathrm{Br}]\right.$.

Identification code

Empirical formula

Formula weight

shelx

Temperature

Wavelength

Crystal system

Space group

Unit cell dimensions

$\mathrm{C}_{14} \mathrm{H}_{24} \mathrm{BrN}_{4} \mathrm{PS}_{2}$

423.37

173(2) K

$0.71073 \AA$

Orthorhombic

F d d 2

$\mathrm{a}=20.7880(16) \AA$

$\mathrm{a}=90^{\circ}$.

$\mathrm{b}=12.8659(7) \AA$

$\mathrm{b}=90^{\circ}$.

$\mathrm{c}=14.3885(9) \AA$

$\mathrm{g}=90^{\circ}$.

Volume

Z

3848.3(4) $\AA^{3}$

Density (calculated)

8

Absorption coefficient

$1.461 \mathrm{Mg} / \mathrm{m}^{3}$

$\mathrm{F}(000)$

$2.438 \mathrm{~mm}^{-1}$

1744

Crystal size

Theta range for data collection

$0.14 \times 0.097 \times 0.03 \mathrm{~mm}^{3}$

Index ranges

Reflections collected

Independent reflections

Completeness to theta $=25.242^{\circ}$

Absorption correction

Max. and min. transmission

Refinement method

Data / restraints / parameters

3.444 to $28.159^{\circ}$.

$-27<=\mathrm{h}<=27,-17<=\mathrm{k}<=17,-19<=\mathrm{l}<=19$

24271

$2360[\mathrm{R}(\mathrm{int})=0.1034]$

$99.8 \%$

Semi-empirical from equivalents

0.929 and 0.851

Full-matrix least-squares on $\mathrm{F}^{2}$

2360 / 1 / 105

Goodness-of-fit on $\mathrm{F}^{2}$

1.024

Final R indices [I $>2$ sigma(I)]

$\mathrm{R}$ indices (all data)

Absolute structure parameter

Extinction coefficient

$\mathrm{R} 1=0.0347, \mathrm{wR} 2=0.0643$

$\mathrm{R} 1=0.0549, \mathrm{wR} 2=0.0700$

$0.100(6)$

Largest diff. peak and hole

$\mathrm{n} / \mathrm{a}$

0.369 and -0.231 e. $\AA^{-3}$ 
Figure S12. Thermal ellipsoid plot of $\left[\left({ }^{\mathrm{Me}} \mathrm{NHC}^{\mathrm{B}}\right)_{2} \mathrm{PS}_{2}\right][\mathrm{I}] \bullet \mathrm{MeCN}$. Ellipsoids are drawn using the $30 \%$ probability surface.

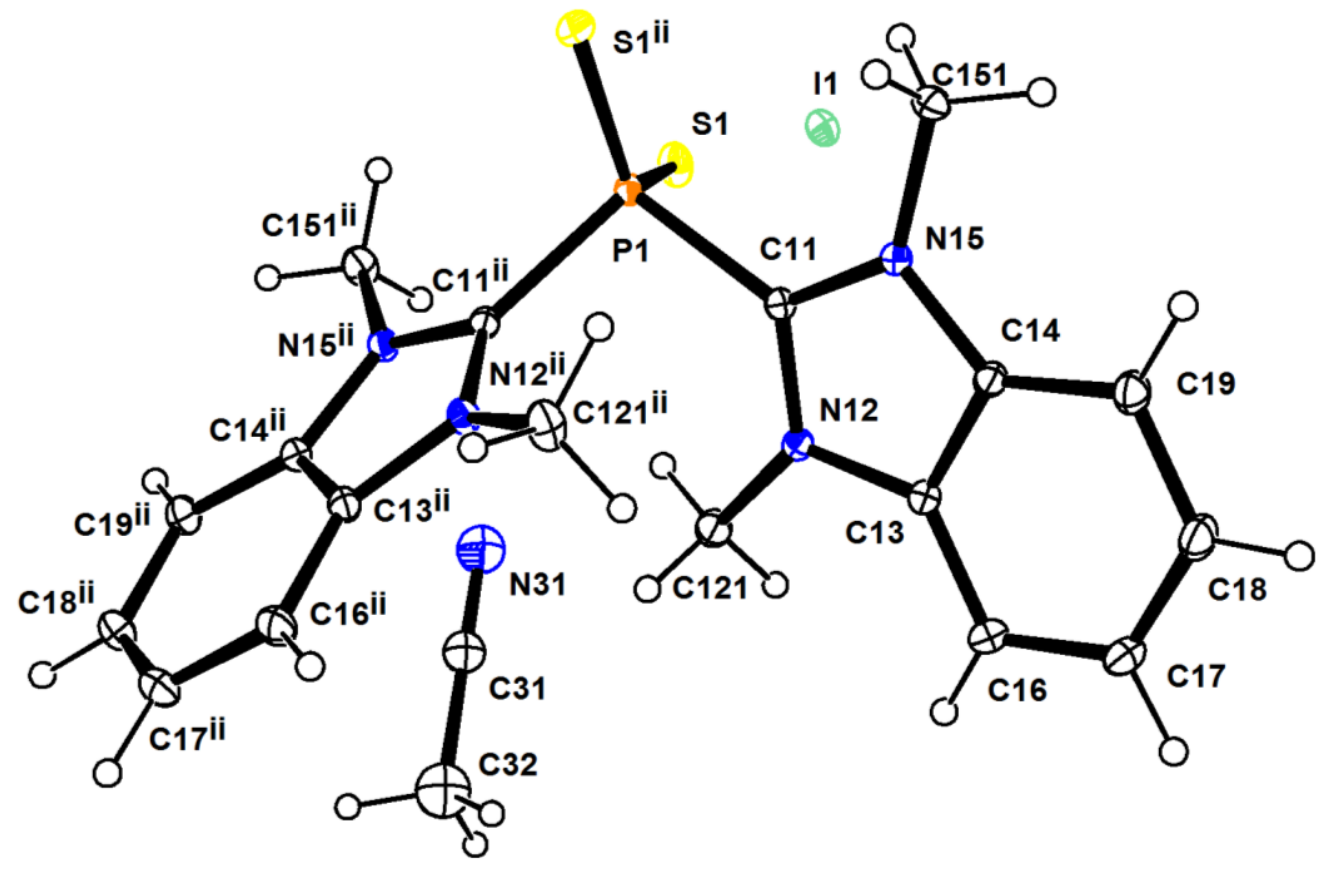

Table S12. Crystal data and structure refinement for $\left[\left({ }^{\mathrm{Me}} \mathrm{NHC}^{\mathrm{B}}\right)_{2} \mathrm{PS}_{2}\right][\mathrm{I}] \cdot \mathrm{MeCN}$.

Identification code

Empirical formula

Formula weight

Temperature

Wavelength

Crystal system

Space group

Unit cell dimensions

Volume

$\mathrm{Z}$

Density (calculated)

Absorption coefficient $\mathrm{F}(000)$

Crystal size

Theta range for data collection

Index ranges

Reflections collected

Independent reflections

Completeness to theta $=25.242^{\circ}$

Absorption correction

Max. and min. transmission

Refinement method

Data / restraints / parameters

Goodness-of-fit on $\mathrm{F}^{2}$

Final R indices [I>2sigma(I)]

$\mathrm{R}$ indices (all data)

Extinction coefficient

Largest diff. peak and hole

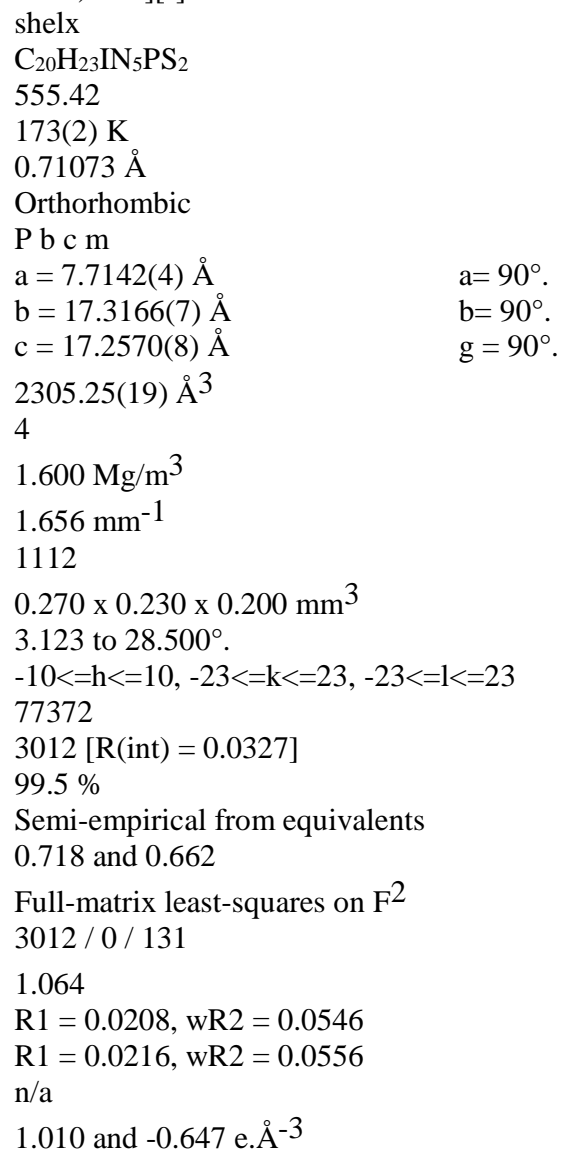

1.010 and -0.647 e. $\AA^{-3}$ 
Figure S13. Thermal ellipsoid plot of $\left[\left({ }^{\mathrm{Me}} \mathrm{NHC}{ }^{\mathrm{Me}}\right)_{2} \mathrm{PH}\right][\mathrm{OTf}]_{2}$. Ellipsoids are drawn using the $30 \%$ probability surface.
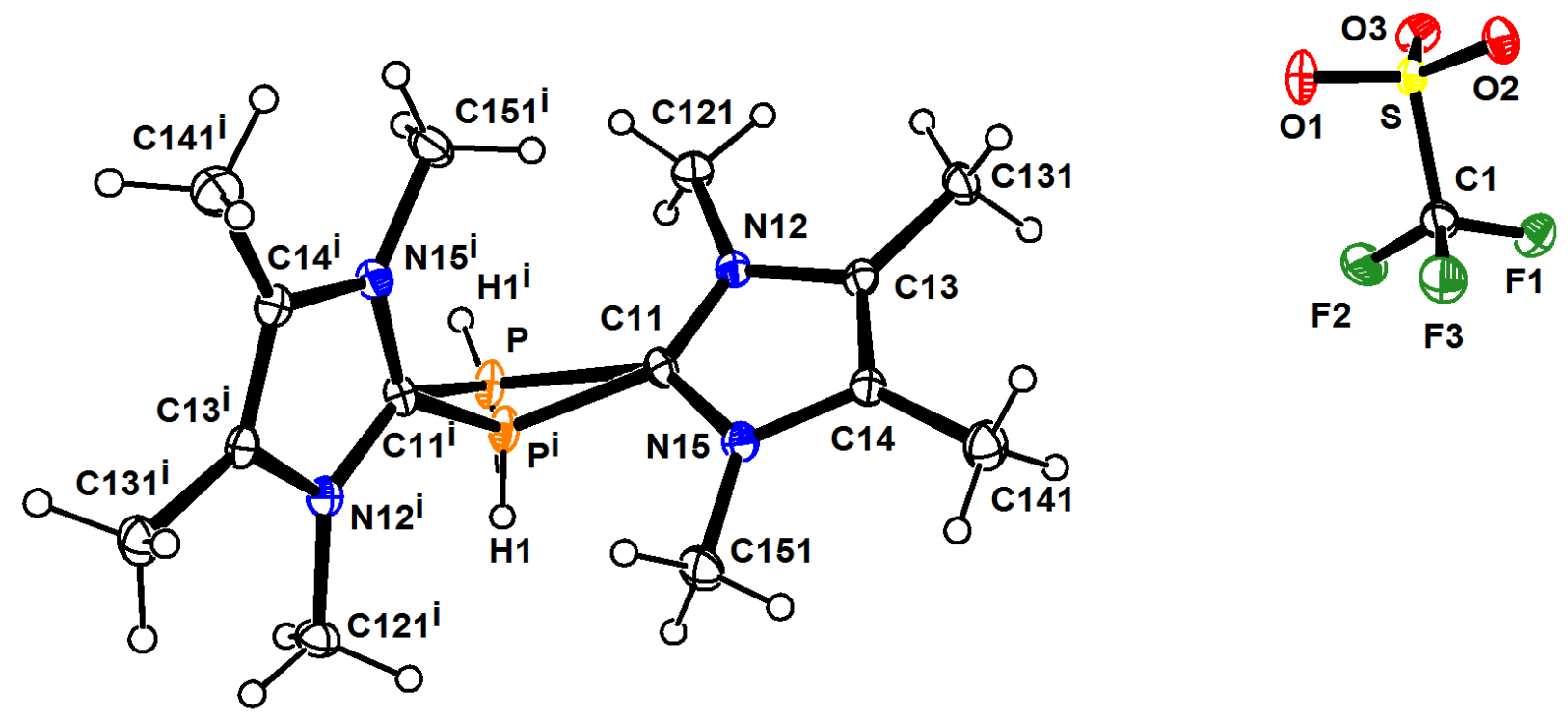

The disorder in the P-H fragments was modeled over 2 sites with a 50:50 occupancy.

Table S13. Crystal data and structure refinement for $\left[\left({ }^{\mathrm{Me}} \mathrm{NHC} \mathrm{Ce}_{2}\right)_{2} \mathrm{PH}\right][\mathrm{OTf}]_{2}$.

Identification code

shelx

Empirical formula

$\mathrm{C}_{16} \mathrm{H}_{25} \mathrm{~F}_{6} \mathrm{~N}_{4} \mathrm{O}_{6} \mathrm{PS}_{2}$

Formula weight

Temperature

Wavelength

Crystal system

Space group

Unit cell dimensions

578.49

173(2) K

$0.71073 \AA$

Monoclinic

\section{$\mathrm{C} 2 / \mathrm{c}$}

$\mathrm{a}=8.8801(15) \AA$

$\mathrm{b}=13.671(2) \AA$

$\mathrm{c}=19.655(3) \AA$

Volume

2367.2(7) $\AA^{3}$

Z

4

Density (calculated)

$1.623 \mathrm{Mg} / \mathrm{m}^{3}$

Absorption coefficient

$0.381 \mathrm{~mm}^{-1}$

$\mathrm{F}(000)$

1192

Crystal size

Theta range for data collection

$0.40 \times 0.30 \times 0.30 \mathrm{~mm}^{3}$

2.089 to $27.496^{\circ}$.

Index ranges

Reflections collected

Independent reflections

Completeness to theta $=25.242^{\circ}$

$-11<=\mathrm{h}<=10,-17<=\mathrm{k}<=17,-24<=\mathrm{l}<=16$

6993

$2540[\mathrm{R}$ (int) $=0.0534]$

$98.7 \%$

Absorption correction

Max. and min. transmission

Semi-empirical from equivalents

0.892 and 0.687

Refinement method

Data / restraints / parameters

Full-matrix least-squares on $\mathrm{F}^{2}$

Goodness-of-fit on $\mathrm{F}^{2}$

2540 / 1 / 167

Final R indices [I $>2 \operatorname{sigma}(\mathrm{I})]$

$\mathrm{R}$ indices (all data)

Extinction coefficient

1.059

$\mathrm{R} 1=0.0778, \mathrm{wR} 2=0.1722$

$\mathrm{R} 1=0.1089, \mathrm{wR} 2=0.1934$

n/a

Largest diff. peak and hole

0.594 and -0.517 e. $\AA^{-3}$ 
Figure S14. Thermal ellipsoid plot of $\left[\left({ }^{\mathrm{Me} N H C}{ }^{\mathrm{B}}\right)_{2} \mathrm{PH}\right][\mathrm{OTf}]_{2} \bullet \mathrm{MeCN}$. Ellipsoids are drawn using the $30 \%$ probability surface.

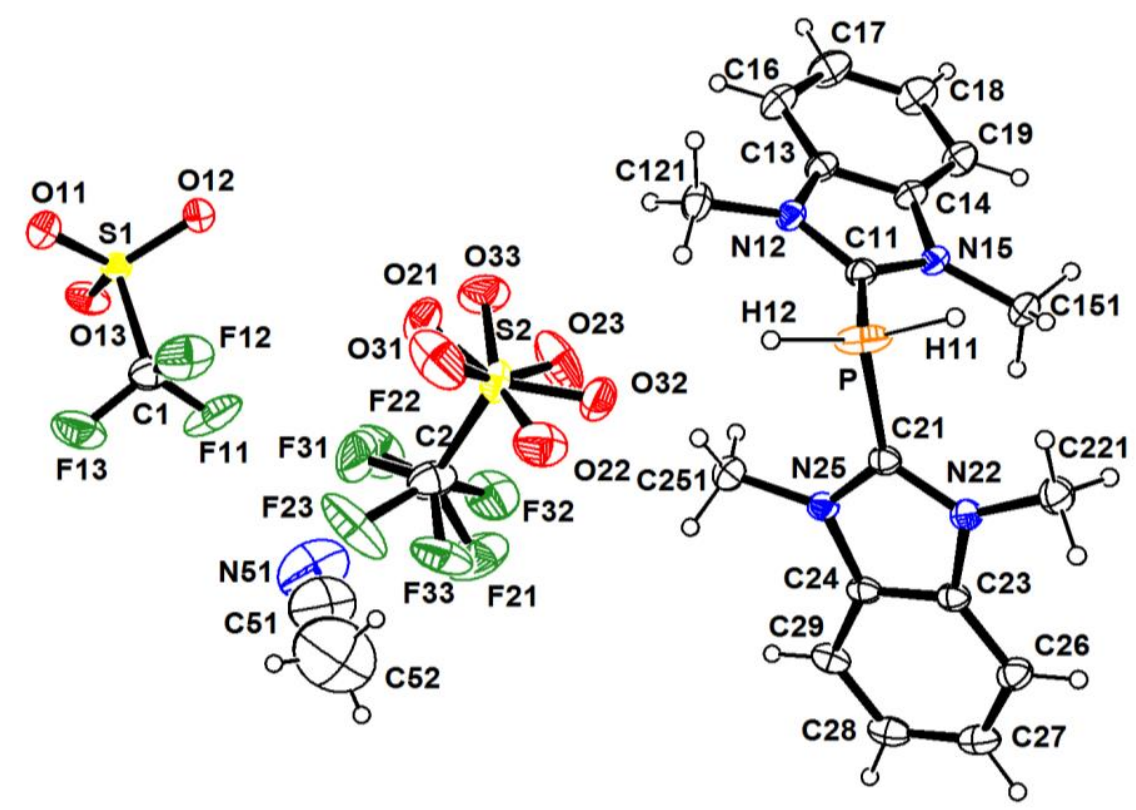

The disorder in the P-H fragments was modeled with a single $\mathrm{P}$-atom position and $2 \mathrm{H}$ atom sites with a 50:50 occupancy. The disordered triflate anion was modeled using 2 sites with shared $\mathrm{C}$ and $\mathrm{S}$ atom positions; the refined ratio of occupancy was $53: 47$.

Table S14. Crystal data and structure refinement for $\left[\left({ }^{\mathrm{Me} N H C}\right)_{2} \mathrm{PH}\right][\mathrm{OTf}]_{2} \bullet \mathrm{MeCN}$.

Identification code

Empirical formula

Formula weight

Temperature

Wavelength

Crystal system

Space group

Unit cell dimensions

Volume

Z

Density (calculated)

Absorption coefficient

$\mathrm{F}(000)$

Crystal size

Theta range for data collection

Index ranges

Reflections collected

shelx

$\mathrm{C}_{22} \mathrm{H}_{24} \mathrm{~F}_{6} \mathrm{~N}_{5} \mathrm{O}_{6} \mathrm{PS}_{2}$

663.55

173(2) K

$1.54178 \AA$

Triclinic

$\mathrm{P}-1$

$\mathrm{a}=8.0203(4) \AA$

$\mathrm{b}=13.8070(6) \AA$

$\mathrm{a}=103.508(2)^{\circ}$.

$\mathrm{c}=13.9727(6) \AA$

$\mathrm{b}=105.014(2)^{\circ}$.

1415.34(11) $\AA^{3}$

$\mathrm{g}=98.757(2)^{\circ}$.

Independent reflections

Completeness to theta $=65.099^{\circ}$

Absorption correction

Max. and min. transmission

Refinement method

Data / restraints / parameters

Goodness-of-fit on $\mathrm{F}^{2}$

2

$1.557 \mathrm{Mg} / \mathrm{m}^{3}$

$3.037 \mathrm{~mm}^{-1}$

680

Final R indices [I $>2 \operatorname{sigma(I)]~}$

$\mathrm{R}$ indices (all data)

Extinction coefficient

$0.14 \times 0.12 \times 0.08 \mathrm{~mm}^{3}$

3.380 to $65.099^{\circ}$.

$-9<=\mathrm{h}<=9,-16<=\mathrm{k}<=16,-16<=1<=16$

20689

$4790[\mathrm{R}(\mathrm{int})=0.0435]$

$99.1 \%$

Semi-empirical from equivalents

0.784 and 0.667

Full-matrix least-squares on $\mathrm{F}^{2}$

4790 / 61 / 446

1.084

$\mathrm{R} 1=0.0509, \mathrm{wR} 2=0.1384$

$\mathrm{R} 1=0.0582, \mathrm{wR} 2=0.1455$

$\mathrm{n} / \mathrm{a}$

Largest diff. peak and hole

0.467 and -0.607 e. $\AA^{-3}$ 
Figure S15. Thermal ellipsoid plot of $\left[\left({ }^{\mathrm{Me}} \mathrm{NHC}^{\mathrm{B}}\right)_{2} \mathrm{PH}\right][\mathrm{I}]_{2} \bullet \mathrm{MeCN}$. Ellipsoids are drawn using the $30 \%$ probability surface.

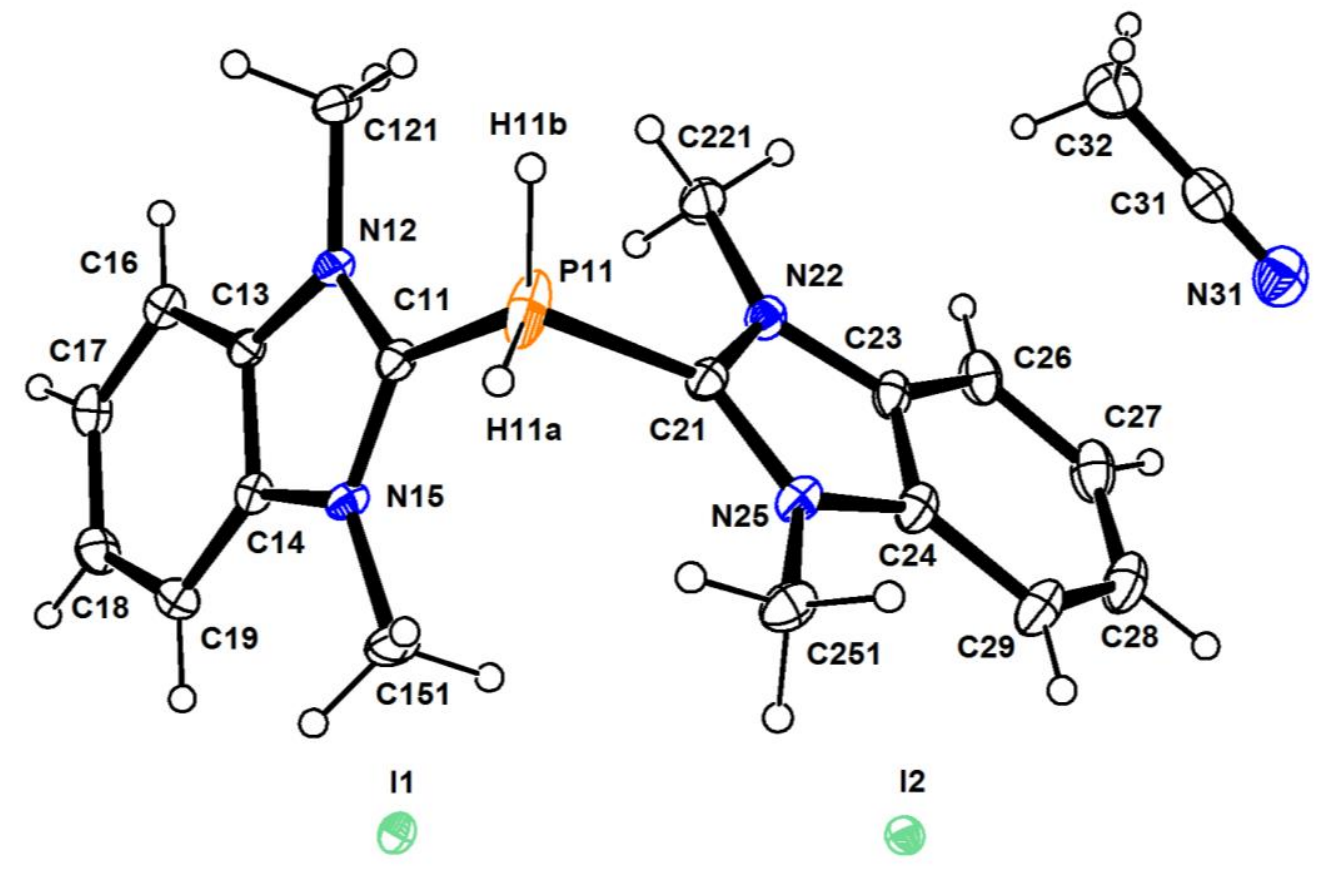

The disorder in the $\mathrm{P}-\mathrm{H}$ fragments was modeled with a single $\mathrm{P}$-atom position and $2 \mathrm{H}$ atom sites with a 50:50 occupancy.

Table S15. Crystal data and structure refinement for $\left[\left({ }^{\mathrm{Me}} \mathrm{NHC}{ }_{2}^{\mathrm{B}}\right)_{2} \mathrm{PH}\right][\mathrm{I}]_{2} \bullet \mathrm{MeCN}$.

Identification code

Empirical formula

Formula weight

Temperature

Wavelength

Crystal system

Space group

Unit cell dimensions

Volume

Z

Density (calculated)

Absorption coefficient

$\mathrm{F}(000)$

Crystal size

Theta range for data collection

Index ranges

Reflections collected

Independent reflections

Completeness to theta $=25.242^{\circ}$

Absorption correction

Max. and min. transmission

Refinement method

Data / restraints / parameters

Goodness-of-fit on $\mathrm{F}^{2}$

Final R indices [I $>2 \operatorname{sigma}(\mathrm{I})$ ]

$\mathrm{R}$ indices (all data)

Extinction coefficient

Largest diff. peak and hole

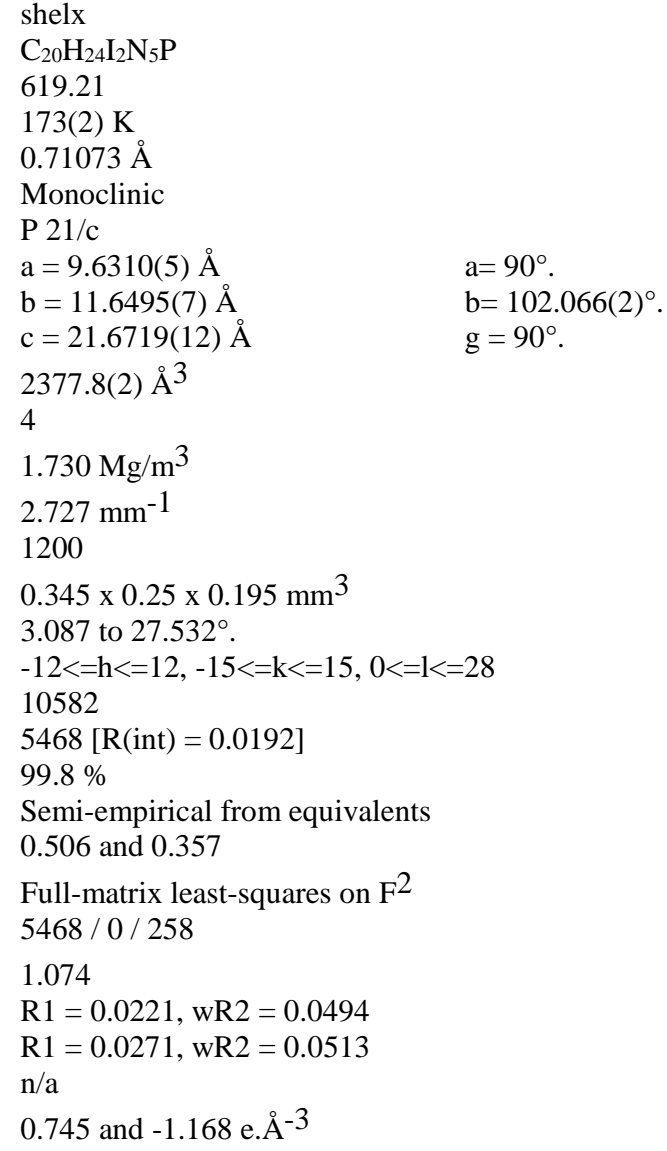


Figure S16. Thermal ellipsoid plot of $\left[\left({ }^{\mathrm{Me}} \mathrm{NHC}^{\mathrm{Me}}\right)_{2} \mathrm{PCH}_{3}\right][\mathrm{OTf}]_{2}$. Ellipsoids are drawn using the $30 \%$ probability surface.

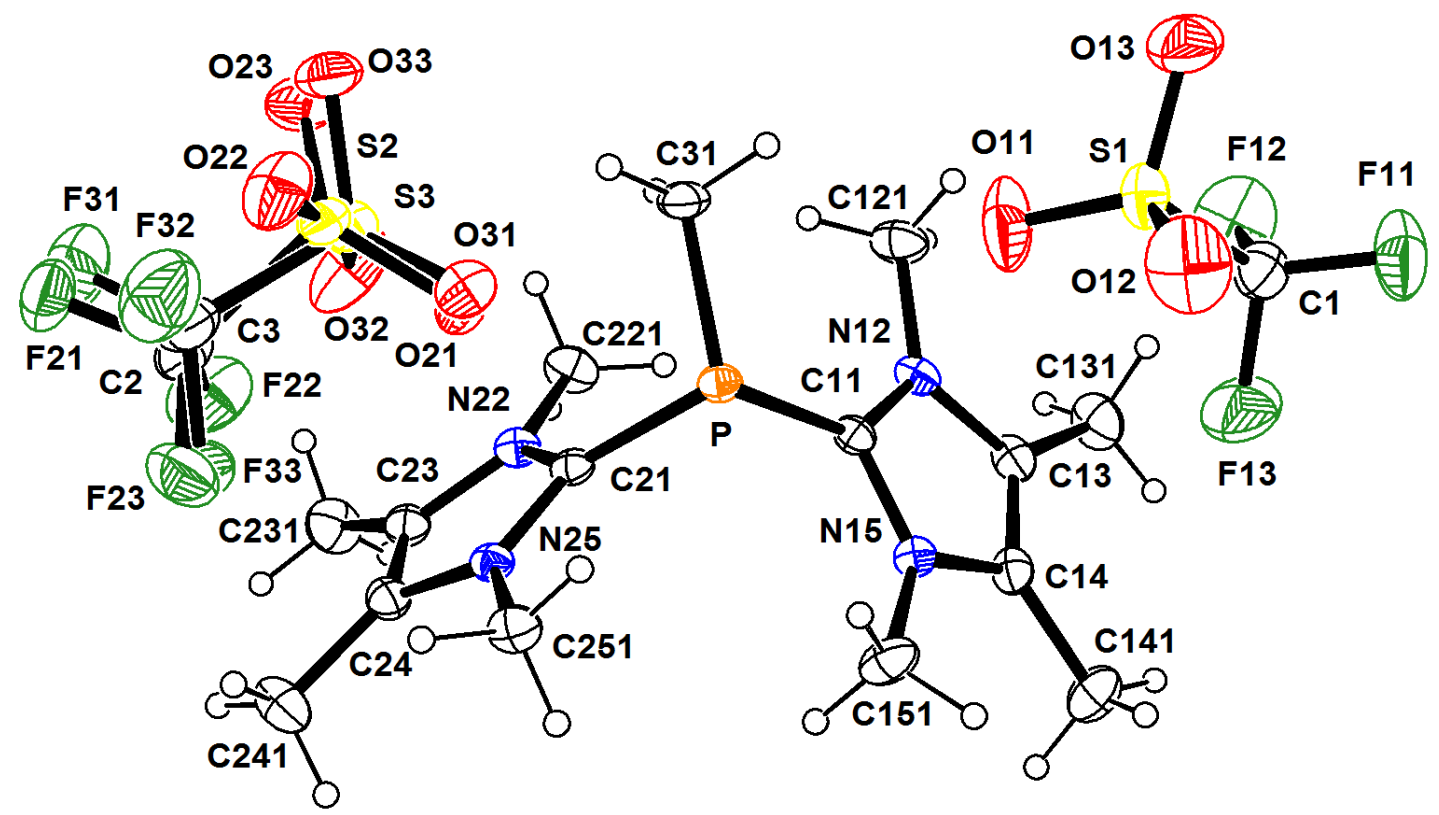

The disordered triflate anion was modeled using 2 sites; the refined ratio of occupancy was 78:22.

Table S16. Crystal data and structure refinement for $\left[\left(\mathrm{Me}^{\mathrm{NHC}}{ }^{\mathrm{Me}}\right)_{2} \mathrm{PCH}_{3}\right][\mathrm{OTf}]_{2}$.

Identification code

Empirical formula

Formula weight

shelx

Temperature

Wavelength

Crystal system

Space group

Unit cell dimensions

Volume

Z

Density (calculated)

Absorption coefficient

$\mathrm{F}(000)$

$\mathrm{C}_{17} \mathrm{H}_{27} \mathrm{~F}_{6} \mathrm{~N}_{4} \mathrm{O}_{6} \mathrm{PS}_{2}$

592.51

243(2) K

$0.71073 \AA$

Monoclinic

$\mathrm{P} 21 / \mathrm{c}$

$\mathrm{a}=8.2808(6) \AA$

$\alpha=90^{\circ}$.

$\mathrm{b}=14.1563(10) \AA$

$\beta=99.477(2)^{\circ}$.

$\mathrm{c}=21.9892(17) \AA$

$\gamma=90^{\circ}$.

Crystal size

Theta range for data collection

Index ranges

Reflections collected

Independent reflections

Completeness to theta $=25.242^{\circ}$

2542.5(3) $\AA^{3}$

4

$1.548 \mathrm{Mg} / \mathrm{m}^{3}$

$0.357 \mathrm{~mm}^{-1}$

1224

$0.171 \times 0.141 \times 0.110 \mathrm{~mm}^{3}$

2.864 to $26.406^{\circ}$.

$-10<=\mathrm{h}<=10,-17<=\mathrm{k}<=17,-27<=\mathrm{l}<=27$

62066

$5203[\mathrm{R}(\mathrm{int})=0.0636]$

$99.9 \%$

Absorption correction

Max. and min. transmission

Semi-empirical from equivalents

0.961 and 0.904

Refinement method

Data / restraints / parameters

Goodness-of-fit on $\mathrm{F}^{2}$

Full-matrix least-squares on $\mathrm{F}^{2}$

5203 / 66 / 407

Final $\mathrm{R}$ indices [I $>2 \operatorname{sigma}(\mathrm{I})]$

1.096

$\mathrm{R}$ indices (all data)

Extinction coefficient

$\mathrm{R} 1=0.0525, \mathrm{wR} 2=0.1194$

$\mathrm{R} 1=0.0795, \mathrm{wR} 2=0.1359$

$\mathrm{n} / \mathrm{a}$

Largest diff. peak and hole

0.405 and -0.325 e. $\AA^{-3}$ 
Figure S17. Thermal ellipsoid plot of $\left[\left({ }^{\mathrm{Me}} \mathrm{NHC}{ }^{\mathrm{Me}}\right)_{2} \mathrm{PAuCl}\right][\mathrm{BPh} 4] \cdot \mathrm{DCM}$. Ellipsoids are drawn using the $30 \%$ probability surface.
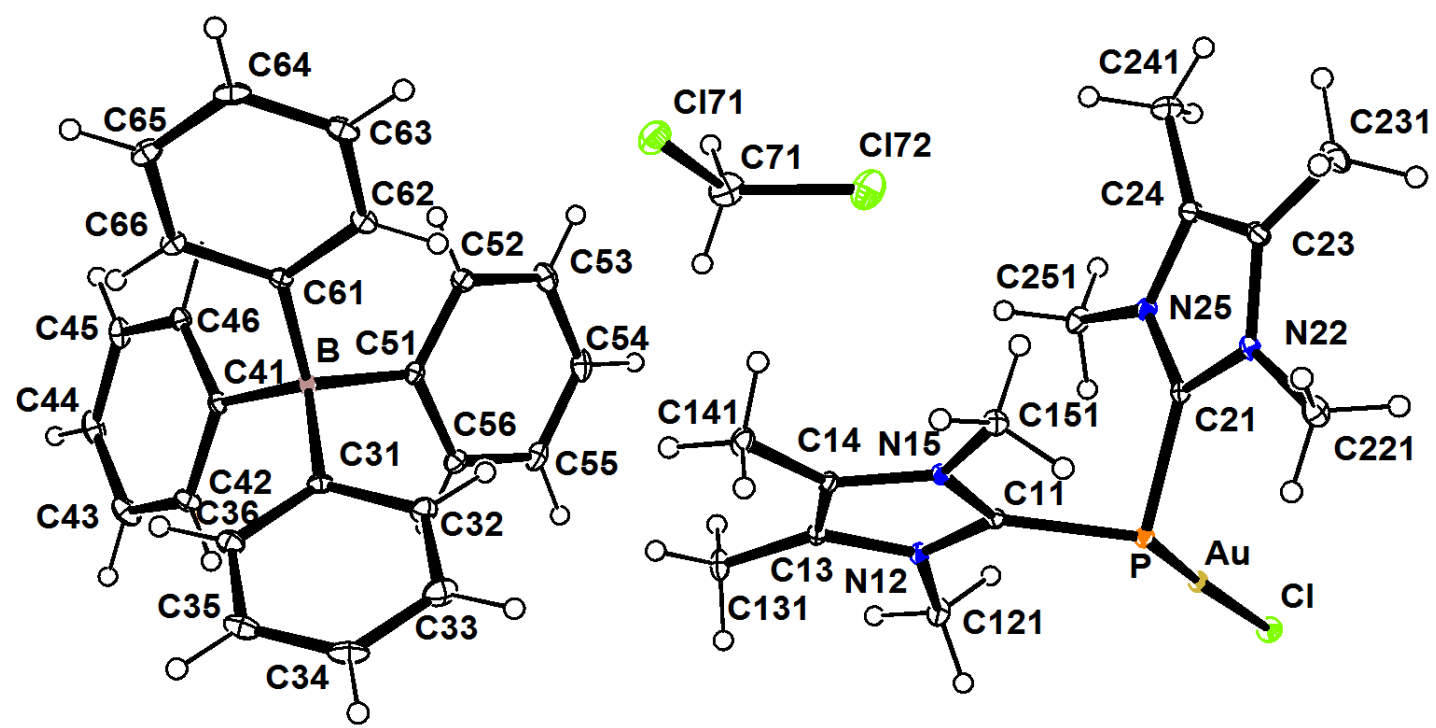

Table S17. Crystal data and structure refinement for $\left[\left({ }^{\mathrm{Me}} \mathrm{NHC}^{\mathrm{Me}}\right)_{2} \mathrm{PAuCl}\right][\mathrm{BPh} 4] \cdot \mathrm{DCM}$.

Identification code

shelx

Empirical formula

$\mathrm{C}_{39} \mathrm{H}_{46} \mathrm{AuBCl}_{3} \mathrm{~N}_{4} \mathrm{P}$

Formula weight

915.89

Temperature

Wavelength

100(2) K

Crystal system

Space group

Unit cell dimensions

$0.71073 \AA$

Triclinic

Volume

Z

Density (calculated)

$\mathrm{P}-1$

$$
\begin{array}{ll}
\mathrm{a}=11.4611(5) \AA & \alpha=100.725(2)^{\circ} . \\
\mathrm{b}=13.1524(5) \AA & \beta=93.311(2)^{\circ} . \\
\mathrm{c}=13.2357(6) \AA & \gamma=92.065(2)^{\circ} .
\end{array}
$$

1954.81(14) $\AA^{3}$

Absorption coefficient

$\mathrm{F}(000)$

Crystal size

Theta range for data collection

Index ranges

Reflections collected

Independent reflections

Completeness to theta $=25.242^{\circ}$

2

$1.556 \mathrm{Mg} / \mathrm{m}^{3}$

$4.042 \mathrm{~mm}^{-1}$

916

$0.170 \times 0.150 \times 0.100 \mathrm{~mm}^{3}$

2.903 to $36.421^{\circ}$.

$-19<=\mathrm{h}<=19,-21<=\mathrm{k}<=21,-22<=\mathrm{l}<=22$

150653

$19023[\mathrm{R}(\mathrm{int})=0.0415]$

$99.8 \%$

Absorption correction

Max. and min. transmission

Refinement method

Data / restraints / parameters

Goodness-of-fit on $\mathrm{F}^{2}$

Semi-empirical from equivalents

0.668 and 0.528

Full-matrix least-squares on $\mathrm{F}^{2}$

19023 / 0 / 450

Final $\mathrm{R}$ indices [I $>2 \operatorname{sigma}(\mathrm{I})]$

1.065

$\mathrm{R}$ indices (all data)

Extinction coefficient

Largest diff. peak and hole

$\mathrm{R} 1=0.0243, \mathrm{wR} 2=0.0406$

$\mathrm{R} 1=0.0349, \mathrm{wR} 2=0.0433$

$\mathrm{n} / \mathrm{a}$

1.497 and -1.083 e. $\AA^{-3}$ 
Figure S18. Thermal ellipsoid plot of $\left[\left({ }^{\mathrm{Me}} \mathrm{NHC}^{\mathrm{Me}}\right)_{2} \mathrm{P}(\mathrm{AuCl})_{2}\right]\left[\mathrm{AuCl}_{2}\right] \cdot \mathrm{DCM} \cdot 2 \mathrm{THF}$. Ellipsoids are drawn using the 30\% probability surface.

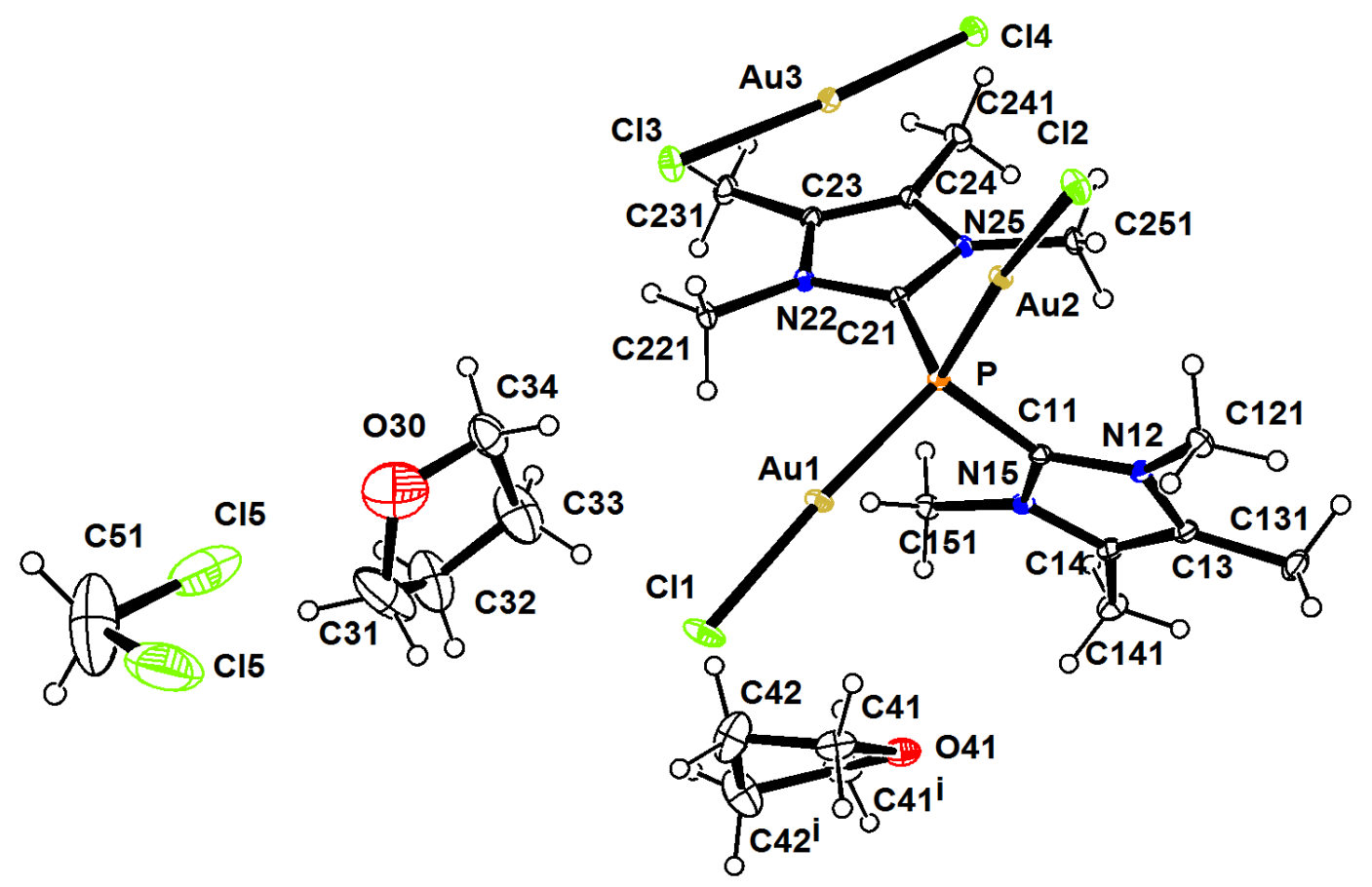

Table S18. Crystal data and structure refinement for $\left[\left({ }^{\mathrm{Me} N H C}{ }^{\mathrm{Me}}\right)_{2} \mathrm{P}(\mathrm{AuCl})_{2}\right]\left[\mathrm{AuCl}_{2}\right] \bullet 0.5 \mathrm{DCM} \bullet 1.5 \mathrm{THF}$.

Identification code

Empirical formula

Formula weight

Temperature

Wavelength

Crystal system

Space group

Unit cell dimensions

Volume

Z

Density (calculated)

Absorption coefficient

$\mathrm{F}(000)$

Crystal size

Theta range for data collection

Index ranges

Reflections collected

Independent reflections

Completeness to theta $=25.242^{\circ}$

Absorption correction

Max. and min. transmission

Refinement method

Data / restraints / parameters

Goodness-of-fit on $\mathrm{F}^{2}$

Final R indices [I $>2 \operatorname{sigma}(\mathrm{I})]$

$\mathrm{R}$ indices (all data)

Extinction coefficient

Largest diff. peak and hole

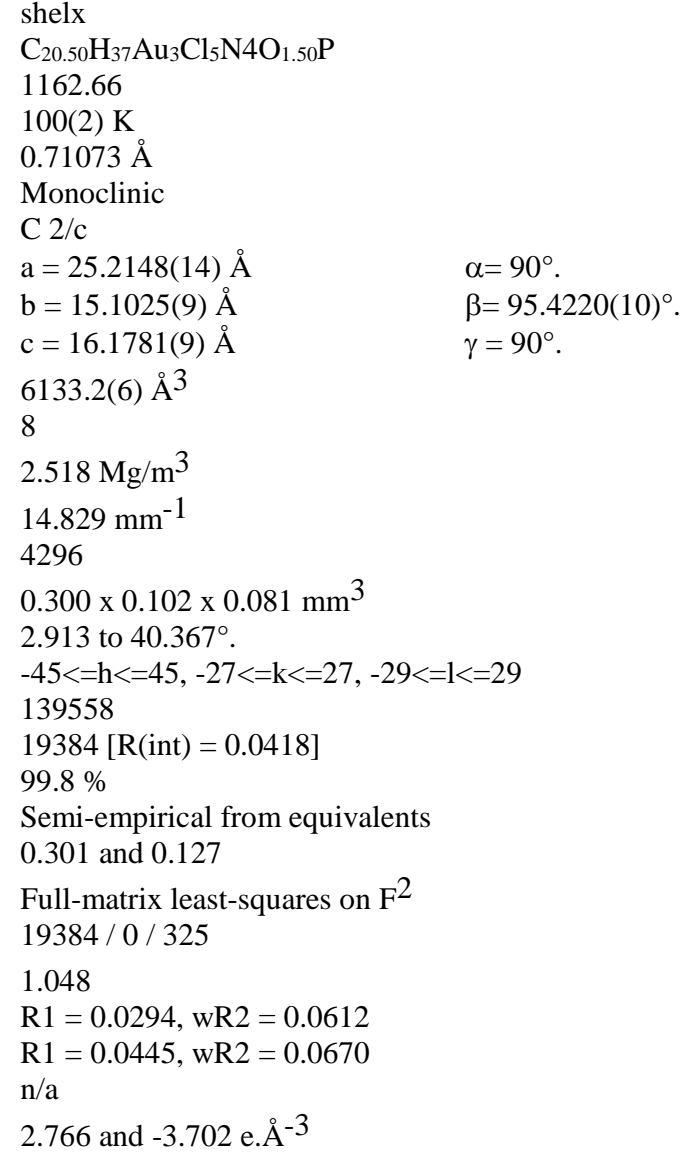




\section{Computational Information}

\section{General Remarks}

Calculations were performed with the Gaussian 09 suite of programs ${ }^{15}$ using Compute Canada's Shared Hierarchical Academic Research Computing Network (SharcNet). Model complexes were fully optimized with no symmetry constraints using the PBE1PBE density functional theory (DFT) method ${ }^{16-18}$ in conjunction with the TZVP basis sets for all atoms ${ }^{19,20}$ using Gaussview. ${ }^{21}$ Frequency calculations were also performed at the same level of theory in order to confirm that the optimized structures were minima on the potential energy hypersurface and to determine thermochemical information. Natural bond orbital (NBO) analyses ${ }^{22}$ to determine orbital contributions, Wiberg Bond Indicies and orbital energies were obtained using the routine included in the Gaussian distributions. ${ }^{23}$ Atoms In Molecules (AIM) analysis was done using AIM2000. ${ }^{24}$

\section{Summary of Calculated Results}

Table S19. Summary of Calculated Results

\begin{tabular}{|c|c|c|c|c|c|c|c|c|}
\hline Model & $\mathbf{N}_{\text {imag }}$ & $\mathbf{E}_{\text {номо }}$ & $\mathbf{E}_{\text {LUMO }}$ & $\mathbf{E}_{\mathrm{H}-\mathrm{L}}$ & $\mathbf{L P}_{(\mathrm{p})}(\mathrm{NBO})^{\mathrm{a}}$ & $\mathbf{E}_{\text {deloc. }}{ }^{b}$ & $\mathbf{R}_{\text {C-P }}$ & $\mathbf{A}_{\mathrm{C}-\mathrm{P}-\mathrm{C}}$ \\
\hline$\left[\left({ }^{\mathrm{Me}} \mathrm{NHC}{ }^{\mathrm{Me}}\right)_{2} \mathrm{P}\right]^{+}$ & 0 & -8.17 & -3.67 & 4.50 & 1.52752 & $\begin{array}{l}61.77 \\
61.74\end{array}$ & $\begin{array}{l}1.8115 \\
1.8125\end{array}$ & 99.30 \\
\hline$\left[\left({ }^{\mathrm{Et}} \mathrm{NHC}{ }^{\mathrm{Me}}\right)_{2} \mathbf{P}\right]^{+}$ & 0 & -7.95 & -3.72 & 4.23 & 1.53866 & $\begin{array}{l}62.23 \\
62.42\end{array}$ & $\begin{array}{l}1.8157 \\
1.8156\end{array}$ & 100.38 \\
\hline$\left[\left({ }^{\mathrm{iPr}} \mathbf{N H C} \mathrm{Cl}^{\mathrm{Me}}\right)_{2} \mathbf{P}\right]^{+}$ & 0 & -7.83 & -3.50 & 4.33 & 1.62174 & $\begin{array}{l}31.99 \\
32.01\end{array}$ & $\begin{array}{c}1.8269 \\
1.8269\end{array}$ & 100.19 \\
\hline
\end{tabular}

${ }^{\text {a }}$ Number of lone pairs assigned to the phosphorus atom in the lowest energy configuration determined by the NBO analysis. ${ }^{b}$ Stabilization energy associated with delocalization of the $\pi$-lone pair on P with the adjacent $\pi$ bonds as determined by the NBO analysis.

\section{$\left[\left({ }^{\mathrm{Me}} \mathrm{NHC}^{\mathrm{Me}}\right)_{2} \mathbf{P}\right]^{+}$}

Figure S19. Optimized geometry of $\left[\left({ }^{\mathrm{Me}} \mathrm{NHC}^{\mathrm{Me}}\right)_{2} \mathrm{P}\right]^{+}$

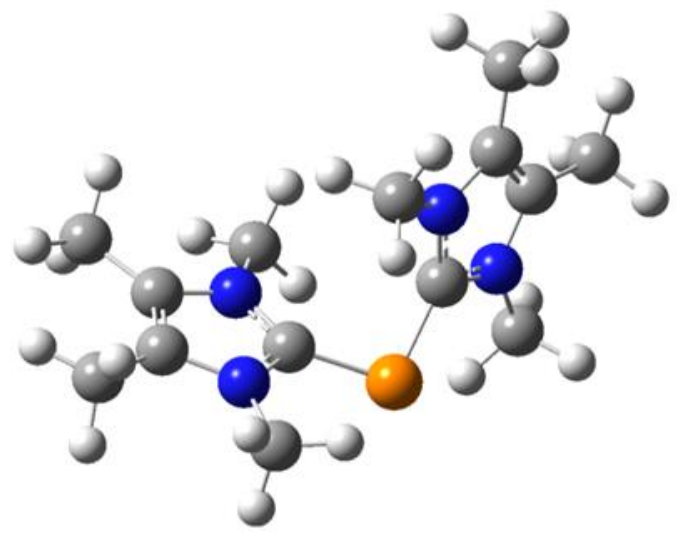

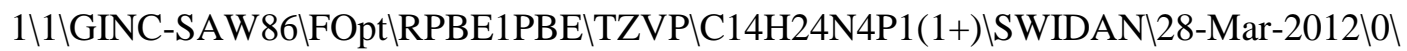


\\# PBE1PBE/TZVP scf=tight opt freq pop=(full,nboread) test $\ O$ Optimizati on of PNHC2Me4\11,1\P,-1.5815972339,0.9579207743,-2.124327726\C,-1.567 0621167,-0.558190126,-1.1330196994lN,-1.626782523,-1.7987556554,-1.668 837066\N,-1.6670983263,-0.7347671122,0.2058958347\C,-1.7682640185,-2.7 528866401,-0.6742540058\C,-1.7775053272,-2.0850595076,0.5095599985\C,$1.5868464314,-2.0874710145,-3.0874934918 \mid C,-1.8740505804,-4.2004967147$ ,-0.9709307985\C,-1.8933173281,-2.5863809299,1.8992518988\C,-1.7739196 $911,0.3383299559,1.1681494911 \backslash \mathrm{C},-0.3912361401,1.9396421566,-1.17518061$ $1 \backslash \mathrm{N},-0.5280479954,3.2673497431,-0.9558840367 \backslash \mathrm{N}, 0.8355873126,1.61693464$ 01,-0.7012097073\C,0.6075728158,3.7809134803,-0.350775056\C,1.46203494 $46,2.7385636808,-0.1748444695 \backslash \mathrm{C},-1.6856379288,4.0512272278,-1.33412399$ 79\C,0.7442304649,5.2148793518,-0.0045112974\C,2.8154442571,2.69146498 $54,0.4268992969 \backslash \mathrm{C}, 1.4526432399,0.3176316387,-0.842485122 \backslash \mathrm{H},-1.11767093$ 26,-1.2439263392,-3.5951450784\Н,-2.5943164392,-2.2225522972,-3.485380 6525\H,-1.0061742357,-2.9923233291,-3.261013131\H,-0.9675477962,-4.588 $5168318,-1.4441553452 \backslash \mathrm{H},-2.7157936063,-4.4132050185,-1.6352289941 \backslash \mathrm{H},-2$ $.0309125381,-4.762202022,-0.0511784598 \backslash \mathrm{H},-1.0611069028,-2.2502456254,2$ $.523486657 \mathrm{VH},-1.888038276,-3.6754684361,1.904099283 \backslash \mathrm{H},-2.8233412658,-2$ $.2623359241,2.3754632404 \backslash \mathrm{H},-0.7925235045,0.6683837957,1.5160802317 \backslash \mathrm{H},-$ $2.3556128149,-0.0016390582,2.022631845 \backslash \mathrm{H},-2.2860863883,1.1754469013,0$. 6925345418\H,-1.5170829417,4.5597822717,-2.2851925787\H,-2.5355426651, $3.3762816845,-1.4413064457 \backslash \mathrm{H},-1.8958078373,4.7902021606,-0.5622418696 \backslash$ H,1.7316724987,5.4084974316,0.4120569954\H,0.0052699669,5.5287009278,0 $.7382544889 \backslash \mathrm{H}, 0.6262633546,5.8528242327,-0.8843787298 \backslash \mathrm{H}, 3.0717819082,3$ $.6643181128,0.8440442669 \backslash \mathrm{H}, 2.8749277814,1.9597729093,1.2370331355 \backslash \mathrm{H}, 3$. $583208702,2.4399239632,-0.3106483378 \backslash \mathrm{H}, 2.5330685076,0.4364483367,-0.89$ 
40111844\Н,1.2077865195,-0.3392788462,-0.0048479177\Н,1.0942211518,-0.

1331274246,-1.7685814355।IVersion=EM64L-G09RevC.01 \State=1-A\HF=-1107.

3294502 \RMSD=4.206e-09\RMSF=9.703e-06\Dipole $=0.5705689,-0.2526522,0.91$

85681\Quadrupole=-9.4131957,22.2130255,-12.7998298,10.7579369,0.538323

3,3.1060508\PG=C01 [X(C14H24N4P1)]1\@

Zero-point correction $=\quad 0.373352($ Hartree/Particle $)$

Thermal correction to Energy $=\quad 0.395877$

Thermal correction to Enthalpy= $\quad 0.396822$

Thermal correction to Gibbs Free Energy $=\quad 0.322556$

Sum of electronic and zero-point Energies $=\quad-1106.956098$

Sum of electronic and thermal Energies $=\quad-1106.933573$

Sum of electronic and thermal Enthalpies $=\quad-1106.932629$

Sum of electronic and thermal Free Energies $=\quad-1107.006894$

NBO Results:

1. (1.96125) BD ( 1) P 1 - C 2

(34.28\%) $0.5855^{*} \mathrm{P} \quad 1 \mathrm{~s}(15.18 \%) \mathrm{p} 5.55(84.20 \%) \mathrm{d} 0.04(0.62 \%)$

$\begin{array}{lllll}0.0000 & -0.0001 & 0.3873 & 0.0425 & -0.0003\end{array}$

$-0.0001-0.6952-0.0446-0.0018 \quad 0.0001$

$\begin{array}{lllll}0.5887 & 0.0131 & 0.0001 & -0.0001 & 0.0993\end{array}$

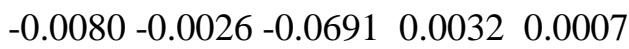

$-0.0074-0.0365$

$(65.72 \%) \quad 0.8107 *$ C 2 s( 39.12\%)p 1.56( 60.85\%)d 0.00( 0.03\%)

$0.0000 \quad 0.6248-0.0271-0.00820 .0001$

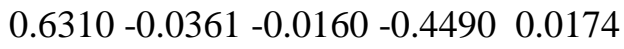

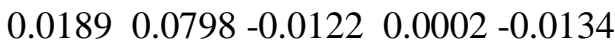


$0.0027-0.0010 \quad 0.0013-0.0094$

2. (1.96124) BD ( 1) P 1 - C 11

(34.27\%) $0.5854 * \mathrm{P} \quad 1 \mathrm{~s}(15.18 \%) \mathrm{p} 5.55(84.21 \%) \mathrm{d} 0.04(0.62 \%)$

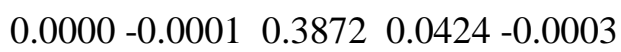

$\begin{array}{lllll}0.0001 & 0.6951 & 0.0446 & 0.0018 & 0.0001\end{array}$

$\begin{array}{lllll}0.5888 & 0.0131 & 0.0001 & 0.0001 & -0.0998\end{array}$

$\begin{array}{lllll}0.0080 & 0.0026 & 0.0691 & 0.0031 & -0.0007\end{array}$

$-0.0075-0.0365$

(65.73\%) $0.8107 *$ C $11 \mathrm{~s}(39.12 \%)$ p $1.56(60.85 \%) d 0.00(0.03 \%)$

$0.0000 \quad 0.6248-0.0271-0.00820 .0001$

$\begin{array}{lllll}-0.6311 & 0.0361 & 0.0159 & -0.4489 & 0.0174\end{array}$

$0.0189-0.0796 \quad 0.0122-0.00020 .0134$

$\begin{array}{lll}0.0027 & 0.0010 & 0.0013-0.0094\end{array}$

72. (1.92712) LP (1) P $1 \quad$ s( 69.93\%)p 0.43(30.03\%)d 0.00( $0.04 \%)$

$\begin{array}{lllll}0.0000 & -0.0005 & 0.8361 & -0.0150 & 0.0001\end{array}$

$\begin{array}{lllll}0.0000 & 0.0001 & 0.0000 & 0.0000 & -0.0004\end{array}$

$\begin{array}{lllll}-0.5480 & 0.0002 & 0.0001 & 0.0000 & 0.0001\end{array}$

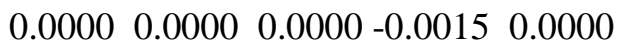

0.01660 .0103

73. (1.52752) LP (2) P $1 \quad$ s( $0.00 \%)$ p $1.00(99.80 \%) d 0.00(0.20 \%)$

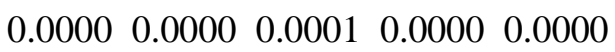

$\begin{array}{lllll}0.0000 & 0.1393 & 0.0111 & 0.0063 & 0.0000\end{array}$

$\begin{array}{lllll}0.0004 & 0.0000 & 0.0000 & 0.0000 & 0.9887\end{array}$

$\begin{array}{lllll}-0.0290 & -0.0002 & 0.0198 & 0.0000 & 0.0399\end{array}$

0.00000 .0000

$\left[\left({ }^{\mathrm{Et}} \mathrm{NHC}^{\mathrm{Me}}\right)_{2} \mathbf{P}\right]_{+}$ 
Figure S20. Optimized geometry of $\left[\left({ }^{\mathrm{Et}} \mathrm{NHC} \mathrm{C}^{\mathrm{Me}}\right)_{2} \mathrm{P}\right]^{+}$

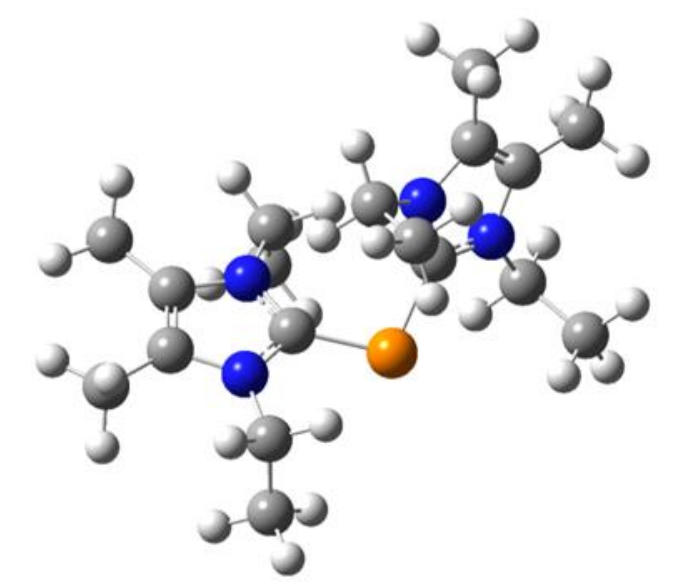

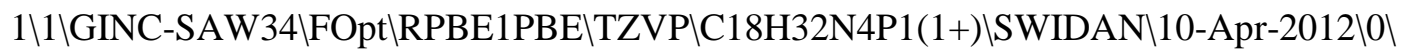

\\# PBE1PBE/TZVP scf=tight opt freq pop=(full,nboread) test $\ O$ optimizati

on of PNHC2Et2Me2\1,1\P,-1.4783000023,0.9133328662,-1.9038477919\C,-1

$.4395514839,-0.6170817564,-0.9275856313 \backslash \mathrm{N},-1.4852346868,-1.8428208298$,

$-1.4993101302 \backslash \mathrm{N},-1.5255228143,-0.8320493087,0.4076108062 \backslash \mathrm{C},-1.61361391$

$3,-2.826475159,-0.5318375991 \backslash C,-1.6261435661,-2.1910891452,0.670017950$

2\C,-1.6723275242,-4.2712111771,-0.8580815054\H,-0.7414765091,-4.62596

65846,-1.3097377097\Н,-2.4879632166,-4.4983443622,-1.5488246065\H,-1.8

$381216843,-4.8525285262,0.0479632403 \backslash \mathrm{C},-1.6999480631,-2.7395683805,2.0$

455873518\H,-2.5523860236,-2.3423290034,2.6020779861\Н,-0.79367082,-2. 5213693947,2.6179092657\H,-1.8093828971,-3.8225680565,2.008790556\C,-0 $.274326679,1.9165629216,-0.9871824729\lfloor\mathrm{~N},-0.4546315194,3.2388942186,-0$. $7627506609 \backslash \mathrm{N}, 0.9757805776,1.6369152412,-0.545052492 \backslash \mathrm{C}, 0.6803976299,3.7$ 952675203,-0.19501915\C,1.5767811574,2.7840688159,-0.0460625178\C,0.77 $22130886,5.2266222448,0.1790283387 \backslash \mathrm{H}, 0.0646231665,5.4878592287,0.97112$ 08704\H,0.5780682422,5.882000115,-0.6734873146\H,1.7721655494,5.453537 $8906,0.5463560327 \backslash \mathrm{C}, 2.9376552732,2.7859885083,0.5425615946 \backslash \mathrm{H}, 3.6920244$ $523,2.4292229732,-0.1629822997 \backslash \mathrm{H}, 2.9925554731,2.1604899743,1.438238605$ 
$\backslash \mathrm{H}, 3.2152719326,3.797960485,0.8342117232 \backslash \mathrm{C},-1.448250185,-2.0859152081$, $-2.9364862424 \backslash \mathrm{C},-2.8239286929,-2.0584726457,-3.576181874 \backslash \mathrm{H},-0.96127654$ 74,-3.0496177859,-3.0903084928\H,-0.8129173053,-1.3075249265,-3.365660 $9785 \backslash \mathrm{H},-2.7345365564,-2.2645475378,-4.6444489151 \backslash \mathrm{H},-3.4868550989,-2.80$ 89534673,-3.141073376\H,-3.289227645,-1.0776620713,-3.4603130552\C,-1. 7364002106,0.2005334522,1.4159882008\C,-3.203444978,0.5580380698,1.571 3274728\Н,-1.1545235027,1.074138563,1.1298801218\H,-1.3190775231,-0.17 09945142,2.352741427\H,-3.3165756147,1.3304258977,2.3346598677\Н,-3.60 $61407529,0.9387373102,0.6312142404 \backslash \mathrm{H},-3.7960233874,-0.3064725355,1.876$ 3745959\C,-1.6613052417,3.9790562106,-1.1120261314\C,-1.6329874533,4.5 $20887483,-2.5290979729 \backslash \mathrm{H},-1.7702211008,4.7842467049,-0.3844801941 \backslash \mathrm{H},-2$ $.4967456055,3.2863829257,-0.9855193 \backslash \mathrm{H},-2.5502456504,5.079227733,-2.725$ 4296554\Н,-0.7872249939,5.192846167,-2.6877569229\Н,-1.5706535918,3.70 92834257,-3.2566465926\C,1.6935133894,0.3889248533,-0.7792947105\C,2.3 717757388,0.3647218731,-2.1370574894\H,0.9833286569,-0.4301761258,-0.6 $879746413 \backslash \mathrm{H}, 2.4194874306,0.2762132506,0.0268982246 \backslash \mathrm{H}, 2.8969411794,-0.5$ 830969738,-2.270842089\Н,1.6344704718,0.4676817045,-2.9349897541\Н,3.1 013577507,1.170835928,-2.2344396821॥Version=EM64L-G09RevC.01 IState=1$\mathrm{A} \backslash \mathrm{HF}=-1264.4475009 \backslash \mathrm{RMSD}=2.994 \mathrm{e}-09 \backslash \mathrm{RMSF}=5.261 \mathrm{e}-06 \backslash \mathrm{Dipole}=0.6723696,-0.2$ 865361,1.0252722\Quadrupole=-9.0442525,20.7401902,-11.6959377,9.679781 ,0.7794125,2.7348101\PG=C01 [X(C18H32N4P1)]川@ Zero-point correction $=\quad 0.487597($ Hartree/Particle $)$ Thermal correction to Energy= $\quad 0.515406$ Thermal correction to Enthalpy $=\quad 0.516350$ Thermal correction to Gibbs Free Energy= $\quad 0.428920$ Sum of electronic and zero-point Energies $=\quad-1263.959904$ 
Sum of electronic and thermal Energies $=\quad-1263.932095$

Sum of electronic and thermal Enthalpies $=\quad-1263.931151$

Sum of electronic and thermal Free Energies $=\quad-1264.018581$

NBO Results:

1. (1.96013) BD ( 1) P 1 - C 2

(34.42\%) $0.5867 * \mathrm{P} 1 \mathrm{~s}(15.48 \%) \mathrm{p} 5.42(83.91 \%) \mathrm{d} 0.04(0.61 \%)$

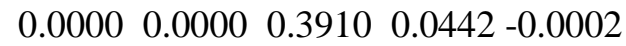

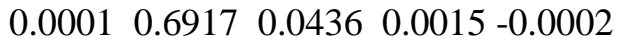

$-0.5858-0.0128-0.0011-0.00010 .1241$

$-0.0086-0.0028-0.0682-0.0031-0.0014$

$-0.0069-0.0365$

(65.58\%) $\quad 0.8098 *$ C 2 s(39.26\%)p 1.55(60.72\%)d 0.00( 0.03\%)

$0.0000 \quad 0.6259-0.0253-0.01140 .0000$

$\begin{array}{lllll}-0.6401 & 0.0339 & 0.0162 & 0.4361 & -0.0179\end{array}$

$-0.01750 .0712-0.0121-0.0004-0.0129$

$\begin{array}{llll}-0.0028 & 0.0013 & 0.0017-0.0091\end{array}$

2. (1.96013) BD ( 1) P 1 - C 15

( $34.43 \%) \quad 0.5867 * \mathrm{P} \quad 1 \mathrm{~s}(15.49 \%) \mathrm{p} 5.42(83.91 \%) \mathrm{d} 0.04(0.61 \%)$

$\begin{array}{lllll}0.0000 & 0.0000 & 0.3910 & 0.0442 & -0.0002\end{array}$

$-0.0001-0.6914-0.0436-0.0015-0.0002$

$-0.5865-0.0128-0.0011 \quad 0.0001-0.1220$

$\begin{array}{lllll}0.0086 & 0.0028 & 0.0682 & -0.0034 & 0.0011\end{array}$

$-0.0069-0.0365$

(65.57\%) $0.8098 *$ C 15 s( 39.26\%)p 1.55( 60.72\%)d 0.00( $0.03 \%)$

$0.0000 \quad 0.6259-0.0253-0.01140 .0000$ 


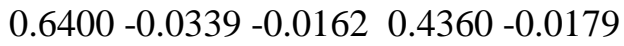

$$
\begin{aligned}
& \begin{array}{lllll}
-0.0175 & -0.0729 & 0.0122 & 0.0005 & 0.0129
\end{array}
\end{aligned}
$$

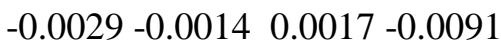

88. (1.92356) LP ( 1) P $1 \quad$ s( 69.34\%)p 0.44(30.62\%)d 0.00( $0.04 \%)$

$0.0000-0.0006 \quad 0.8326-0.0157 \quad 0.0001$

$\begin{array}{lllll}0.0000 & -0.0001 & 0.0000 & 0.0000 & 0.0004\end{array}$

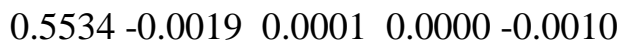

$\begin{array}{lllll}0.0000 & 0.0000 & 0.0000 & 0.0014 & 0.0001\end{array}$

0.01600 .0106

89. (1.53866) LP (2) P $1 \quad$ s( $0.00 \%)$ p $1.00(99.81 \%)$ d $0.00(0.19 \%)$

$$
\begin{array}{lllll}
0.0000 & 0.0000 & 0.0001 & 0.0000 & 0.0000 \\
0.0000 & -0.1722 & -0.0168 & -0.0028 & 0.0000 \\
0.0017 & -0.0001 & 0.0000 & 0.0000 & 0.9836 \\
-0.0254 & -0.0005 & 0.0218 & -0.0001 & -0.0381 \\
0.0001 & 0.0001 & & &
\end{array}
$$

$\left[\left({ }^{i \mathrm{Pr}} \mathrm{NHC} \mathrm{C}^{\mathrm{Me}}\right)_{2} \mathbf{P}\right]^{+}$

Figure S21. Optimized geometry of $\left[\left(^{(\mathrm{iPr}} \mathrm{NHC}^{\mathrm{Me}}\right)_{2} \mathrm{P}\right]^{+}$

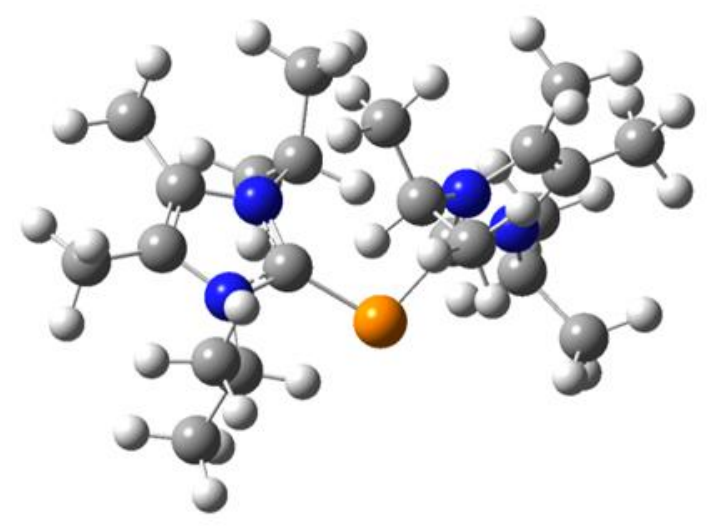

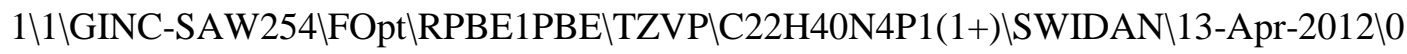
II\# PBE1PBE/TZVP scf=tight opt freq pop=(full,nboread) test $\backslash$ Optimizat 
ion of PNHC2iP2Me2\1,1\P,-1.5688423305,0.9408058494,-2.0411747548\C,$1.5803535587,-0.558759677,-0.9977753803$ WN,-1.5854976091,-1.8152727014, $-1.5078615796 \backslash \mathrm{N},-1.7686358149,-0.6880918445,0.3395624061 \backslash \mathrm{C},-1.78933358$ $12,-2.7361241732,-0.4923583372 \backslash C,-1.8862649143,-2.0305283673,0.6688392$ 488\C,-1.8556429153,-4.2076221761,-0.6804308822\Н,-0.8695507847,-4.647 $6532559,-0.8465411254 \backslash \mathrm{H},-2.4932042778,-4.4826095681,-1.5209472844 \backslash \mathrm{H},-2$ $.2773841294,-4.6729669932,0.2095839265 \backslash C,-2.0203512519,-2.5603948769,2$ $.0495951321 \mathrm{H},-2.821132602,-2.0799514725,2.6122325016 \backslash \mathrm{H},-1.0937827965$, $-2.4446375289,2.6174614863 \backslash \mathrm{H},-2.2446886458,-3.6254257941,2.0107901688 \backslash$ C,-0.2843326772,1.9192044831,-1.1866029373\N,-0.4273885327,3.236423993 $1,-0.8975893571 \backslash \mathrm{N}, 1.0001218632,1.5995806001,-0.888904608 \backslash \mathrm{C}, 0.771614272$ 1,3.7497363434,-0.4288687908\C,1.6620953018,2.7191485178,-0.4063395002 IC,0.9886471132,5.1594569562,-0.0157596686\H,0.5440963749,5.3775587382 $, 0.958095712 \backslash \mathrm{H}, 0.5775153881,5.86391755,-0.7391576561 \backslash \mathrm{H}, 2.0567139966,5$. $360198251,0.0582547891 \backslash \mathrm{C}, 3.0593563297,2.7317898572,0.09635857 \backslash \mathrm{H}, 3.7666$ 09807,2.2900236247,-0.6059902177\Н,3.1509058315,2.1988647838,1.0460954 042\H,3.3743259866,3.7596317811,0.2708793361\C,-2.0715717388,0.4592501 282,1.2145874251\C,-1.1688763177,0.5362599706,2.43435975\C,-3.55496651 $93,0.5077268563,1.5492604613 \backslash \mathrm{H},-1.8570744623,1.3187556001,0.5807738931$ $\backslash \mathrm{H},-0.1161983695,0.4551807749,2.1568616768 \backslash \mathrm{H},-1.3138900215,1.504297309$ 5,2.918343286\H,-1.3936817638,-0.2310457349,3.1749526446\H,-4.15472392 $8,0.5062659196,0.6372969635 \backslash H,-3.8729329738,-0.3265900401,2.1756805317$ $\backslash \mathrm{H},-3.7708103049,1.4280454043,2.0960692502 \backslash \mathrm{C},-1.4413216484,-2.08567423$ 5,-2.9509418202\C,-2.7869300856,-2.3839895236,-3.5925253402\C,-0.37891 01782,-3.1293754786,-3.256965619\Н,-1.0908478384,-1.1248575034,-3.3419 741679\Н,-3.4985987289,-1.5786830579,-3.4012280774\Н,-2.6603365347,-2 . 
4714336678,-4.673453956\Н,-3.2191394913,-3.3199640415,-3.2329020589\H, $0.5372682529,-2.9460503071,-2.6910441334 \mathrm{UH},-0.7114714843,-4.1484603238$ ,-3.0610299541\H,-0.1317555495,-3.0685272906,-4.3184940505\C,1.6169516 $883,0.3238862342,-1.296342366 \backslash \mathrm{C}, 2.3010571486,-0.4029185365,-0.15073738$ $25 \backslash \mathrm{C}, 2.5077508965,0.5182962194,-2.5143223391 \mathrm{H}, 0.7640405667,-0.2742892$ $865,-1.6144402722 \backslash \mathrm{H}, 1.6449164021,-0.4843433029,0.7179224589 \backslash \mathrm{H}, 2.552364$ $21,-1.4144159204,-0.4764303467 \backslash \mathrm{H}, 3.2299072029,0.0760713076,0.158390248$ 5\H,1.9516592427,0.9924357334,-3.3250938584\Н,3.3902355795,1.121903890 5,-2.2987371433\H,2.8554230051,-0.4556309121,-2.8652716419\C,-1.690172 3922,3.9649886795,-1.1231440317\C,-1.6299109725,4.7896590026,-2.398911 9691\C,-2.1433244452,4.7593672763,0.0914781918\Н,-2.4099237016,3.15540 72925,-1.2826858488\Н,-1.3638415642,4.1669079053,-3.2550475279\Н,-2.61 $12651632,5.2270697533,-2.5930549143 \backslash \mathrm{H},-0.9103694269,5.6079895072,-2.32$ 97624411\Н,-2.0662337593,4.1692526995,1.0074901553ไH,-1.588335948,5.68 73391875,0.2270577758\H,-3.1928432463,5.0273102901,-0.0440036839॥Vers ion=EM64L-G09RevC.01\State=1-A $\mathrm{HF}=-1421.5469239 \backslash \mathrm{RMSD}=7.918 \mathrm{e}-09 \backslash \mathrm{RMSF}=5$. 892e-06\Dipole=0.5999065,-0.2457842,0.8945449\Quadrupole=-7.7040385,19 $.8835246,-12.1794861,8.4160565,-2.5733531,3.9286788 \backslash \mathrm{PG}=\mathrm{C} 01$ [X(C22H40N4 P1)]M@ Zero-point correction $=\quad 0.600666($ Hartree/Particle $)$ Thermal correction to Energy= $\quad 0.633620$ Thermal correction to Enthalpy $=\quad 0.634564$ Thermal correction to Gibbs Free Energy $=\quad 0.538089$ Sum of electronic and zero-point Energies $=\quad-1420.946258$

Sum of electronic and thermal Energies $=\quad-1420.913304$

Sum of electronic and thermal Enthalpies $=\quad-1420.912360$ 
Sum of electronic and thermal Free Energies $=\quad-1421.008835$

NBO Results:

1. (1.95670) BD ( 1) P 1 - C 2

(33.98\%) $\quad 0.5829 * \mathrm{P} \quad 1 \mathrm{~s}(15.16 \%) \mathrm{p} 5.55(84.21 \%) \mathrm{d} 0.04(0.63 \%)$

$0.0000-0.0001 \quad 0.38760 .0376-0.0002$

$\begin{array}{llll}0.0000 & 0.6869 & 0.0429 & -0.0007-0.0002\end{array}$

$\begin{array}{lllll}-0.1461 & -0.0121 & -0.0041 & 0.0002 & 0.5889\end{array}$

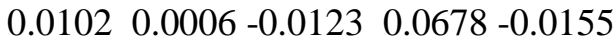

0.02600 .0243

(66.02\%) $0.8125 * \mathrm{C} 2 \mathrm{~s}(38.81 \%) \mathrm{p} 1.58(61.17 \%) \mathrm{d} 0.00(0.03 \%)$

$0.0000 \quad 0.6223-0.0267-0.0108 \quad 0.0000$

$\begin{array}{lllll}-0.6203 & 0.0350 & 0.0133 & 0.1539 & -0.0169\end{array}$

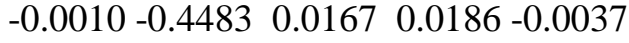

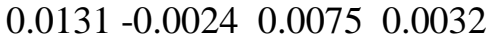

2. (1.95670) BD ( 1) P 1 - C 15

(33.98\%) $0.5829 * \mathrm{P} \quad 1 \mathrm{~s}(15.16 \%) \mathrm{p} 5.55(84.21 \%) \mathrm{d} 0.04(0.63 \%)$

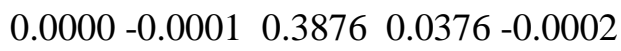

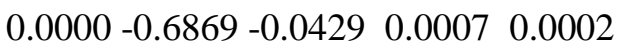

$\begin{array}{lllll}0.1460 & 0.0121 & 0.0041 & 0.0002 & 0.5890\end{array}$

$0.0102 \quad 0.0006-0.0123-0.06780 .0155$

0.02590 .0243

(66.02\%) $0.8125 *$ C 15 s( 38.81\%)p 1.58( 61.17\%)d 0.00( $0.03 \%)$

$0.0000 \quad 0.6223-0.0267-0.0108 \quad 0.0000$

$0.6202-0.0350-0.0133-0.15370 .0169$

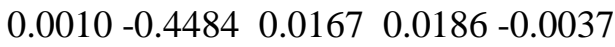


$\begin{array}{llll}-0.0131 & 0.0024 & 0.0075 & 0.0032\end{array}$

104. (1.91928) LP (1) P $1 \quad$ s( 69.89\%)p 0.43(30.07\%)d 0.00( $0.04 \%)$

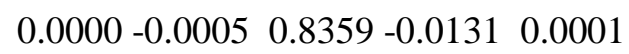

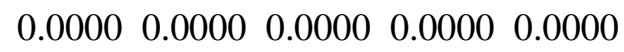

$\begin{array}{llll}0.0001 & 0.0000 & 0.0000 & -0.0004-0.5483\end{array}$

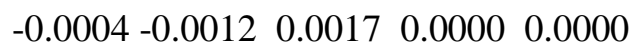

$-0.0015-0.0197$

105. (1.62174) LP( 2) P $1 \quad$ s( $0.00 \%) p$ 1.00( 99.88\%)d 0.00( $0.12 \%)$

$\begin{array}{lllll}0.0000 & 0.0000 & 0.0000 & 0.0000 & 0.0000\end{array}$

$\begin{array}{llll}0.0003 & 0.2078-0.0053-0.0040 & 0.0007\end{array}$

$\begin{array}{lllll}0.9771 & -0.0275 & 0.0002 & 0.0000 & 0.0000\end{array}$

$\begin{array}{lllll}0.0000 & 0.0000 & 0.0000 & 0.0056 & 0.0344\end{array}$

0.00000 .0000 


\section{Cyclic Voltammetry of $\left[\left({ }^{\mathrm{Me}} \mathrm{NHC}^{\mathrm{Me}}\right)_{2} \mathrm{P}\right][\mathrm{OTf}]$}

Figure 22. Voltammogram of a $0.01 \mathrm{M}$ solution of $\left[\left({ }^{\mathrm{Me}} \mathrm{NHC}{ }^{\mathrm{Me}}\right)_{2} \mathrm{P}\right][\mathrm{OTf}]$ in acetonitrile with $0.1 \mathrm{M}\left[n \mathrm{Bu}_{4} \mathrm{~N}\right]\left[\mathrm{PF}_{6}\right]$ as supporting electrolyte at a scan rate of $100 \mathrm{mV} / \mathrm{s}$ and a sensitivity of $100 \mu \mathrm{A} / \mathrm{V}$. A glassy carbon electrode, a platinum wire, and an $\mathrm{Ag} / \mathrm{AgCl}$ electrode were used as the working, auxiliary, and reference electrodes, respectively. The scale in the figure is with respect to $\mathrm{Ag} / \mathrm{AgCl}$ reference electrode.

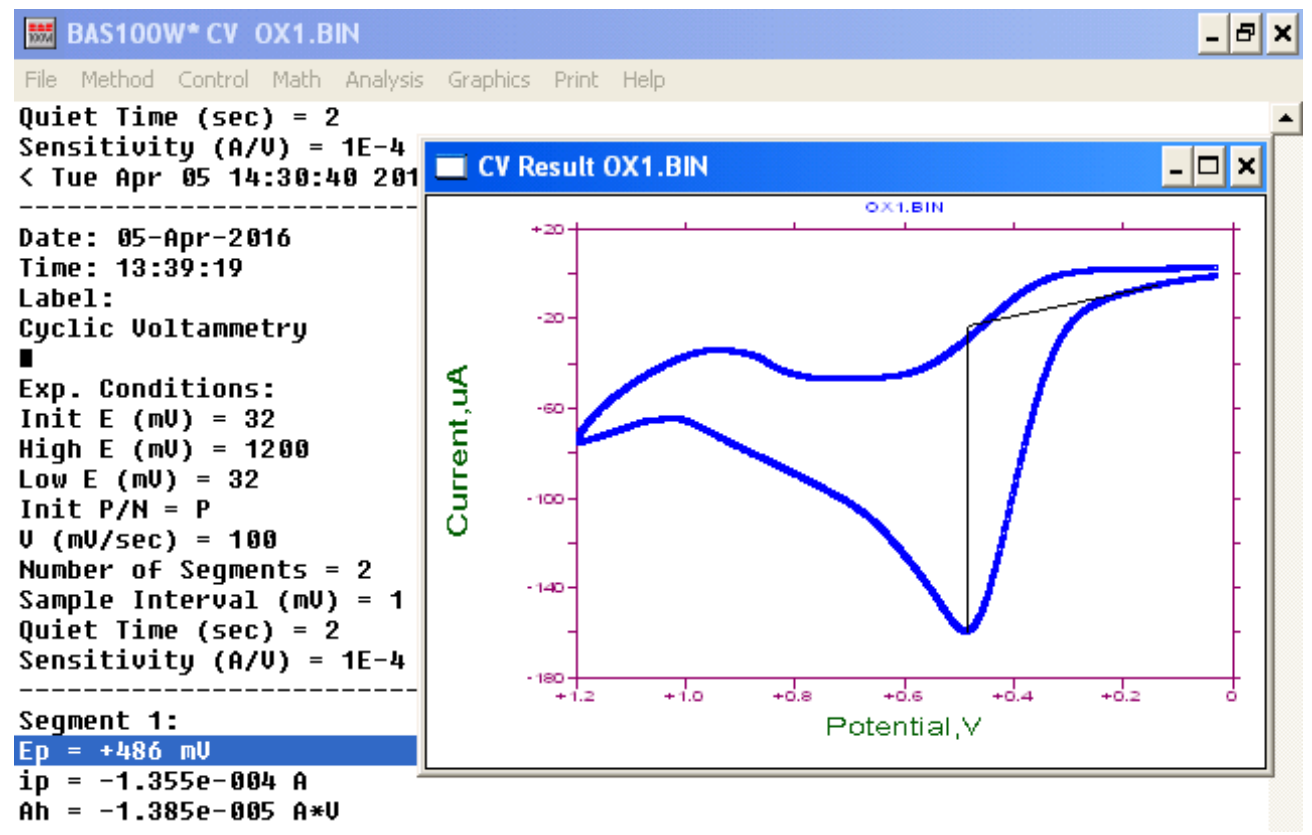




\section{Carbene and $\mathrm{PX}_{3}$ Reaction Results}

\section{1:1 Reaction of ${ }^{\mathrm{Me}} \mathrm{NHC}^{\mathrm{Me}}$ and $\mathrm{PCl}_{3}$}

To a solution of ${ }^{\mathrm{Me}} \mathrm{NHC}{ }^{\mathrm{Me}}(50.0 \mathrm{mg}, 0.403 \mathrm{mmol})$ was added $\mathrm{PCl}_{3}(55.0 \mathrm{mg}, 0.403 \mathrm{mmol})$ in THF (1 mL). Upon addition, a yellow precipitate formed immediately. ${ }^{31} \mathrm{P}\left\{{ }^{1} \mathrm{H}\right\} \mathrm{NMR}(\mathrm{THF}) \delta$ : $115.2(\mathrm{~s}), 218.8\left(\mathrm{~s}, \mathrm{PCl}_{3}\right)$.

\section{1:1 Reaction of ${ }^{\mathrm{Me}} \mathrm{NHC}^{\mathrm{Me}}$ and $\mathrm{PBr}_{3}$}

To a solution of ${ }^{\mathrm{Me}} \mathbf{N H C}{ }^{\mathrm{Me}}(50.0 \mathrm{mg}, 0.403 \mathrm{mmol})$ was added $\mathrm{PBr}_{3}(109 \mathrm{mg}, 0.403 \mathrm{mmol})$ in THF (1 mL). Upon addition, a yellow/orange precipitate formed immediately. ${ }^{31} \mathrm{P}\left\{{ }^{1} \mathrm{H}\right\} \mathrm{NMR}$ (THF) $\delta:-98.0(\mathrm{~s}),-30.3,230.1\left(\mathrm{~s}, \mathrm{PBr}_{3}\right)$.

\section{1:1 Reaction of ${ }^{\mathrm{Me}} \mathrm{NHC}^{\mathrm{Me}}$ and PI3}

To a solution of ${ }^{\mathrm{Me}} \mathbf{N H C}{ }^{\mathrm{Me}}(50.0 \mathrm{mg}, 0.403 \mathrm{mmol})$ was added $\mathrm{PI}_{3}(166 \mathrm{mg}, 0.403 \mathrm{mmol})$ in $\mathrm{THF}$ (1 mL). Upon addition, a yellow/orange precipitate formed immediately. ${ }^{31} \mathrm{P}\left\{{ }^{1} \mathrm{H}\right\} \mathrm{NMR}$ (THF) $\delta$ : $102.8\left(\mathrm{~s}, \mathrm{P}_{2} \mathrm{I}_{4}\right), 174.2\left(\mathrm{~s}, \mathrm{PI}_{3}\right)$.

\section{1:1 Reaction of ${ }^{\mathrm{Et}} \mathrm{NHC}^{\mathrm{Me}}$ and $\mathrm{PCl}_{3}$}

To a solution of ${ }^{\mathbf{E t}} \mathbf{N H C}{ }^{\mathrm{Me}}(50.0 \mathrm{mg}, 0.328 \mathrm{mmol})$ was added $\mathrm{PCl}_{3}(45.0 \mathrm{mg}, 0.328 \mathrm{mmol})$ in THF (1 mL). Upon addition, a yellow precipitate formed immediately. ${ }^{31} \mathrm{P}\left\{{ }^{1} \mathrm{H}\right\} \mathrm{NMR}(\mathrm{THF}) \delta$ : $96.1(\mathrm{~s}),-30.68(\mathrm{~s}), 16.97(\mathrm{~s}), 212.72\left(\mathrm{~s}, \mathrm{PCl}_{3}\right)$.

\section{1:1 Reaction of ${ }^{\mathrm{Et}} \mathrm{NHC}^{\mathrm{Me}}$ and $\mathrm{PBr}_{3}$}


To a solution of ${ }^{\mathbf{E t}} \mathbf{N H C}{ }^{\mathrm{Me}}(50.0 \mathrm{mg}, 0.328 \mathrm{mmol})$ was added $\mathrm{PBr}_{3}(89.0 \mathrm{mg}, 0.328 \mathrm{mmol})$ in THF (1 mL). Upon addition, a dark orange precipitate formed immediately. ${ }^{31} \mathrm{P}\left\{{ }^{1} \mathrm{H}\right\} \mathrm{NMR}$ (THF) $\delta:-96.3(\mathrm{~s}),-30.0(\mathrm{~s}), 17.6(\mathrm{~s}), 225.9\left(\mathrm{~s}, \mathrm{PBr}_{3}\right)$.

\section{1:1 Reaction of ${ }^{\mathrm{Et}} \mathrm{NHC}^{\mathrm{Me}}$ and $\mathrm{PI}_{3}$}

To a solution of ${ }^{\mathbf{E t}} \mathbf{N H C}{ }^{\mathrm{Me}}(50.0 \mathrm{mg}, 0.328 \mathrm{mmol})$ was added $\mathrm{PI}_{3}(135 \mathrm{mg}, 0.328 \mathrm{mmol})$ in $\mathrm{THF}$ $(1 \mathrm{~mL})$. Upon addition, a dark orange precipitate formed immediately. ${ }^{31} \mathrm{P}\left\{{ }^{1} \mathrm{H}\right\} \mathrm{NMR}(\mathrm{THF}) \delta$ : $80.3(\mathrm{~s}),-27.9(\mathrm{~s}), 8.1(\mathrm{~s}), 99.4\left(\mathrm{~s}, \mathrm{P}_{2} \mathrm{I}_{4}\right), 166.9\left(\mathrm{~s}, \mathrm{PI}_{3}\right)$.

\section{1:1 Reaction of ${ }^{\text {iPr }} \mathrm{NHC}^{\mathrm{Me}}$ and $\mathrm{PCl}_{3}$}

To a solution of ${ }^{\text {Pr }}{ }^{-} H^{\mathrm{Me}}(50.0 \mathrm{mg}, 0.277 \mathrm{mmol})$ was added $\mathrm{PCl}_{3}(38.0 \mathrm{mg}, 0.277 \mathrm{mmol})$ in THF (1 mL). Upon addition, a yellow precipitate formed immediately. ${ }^{31} \mathrm{P}\left\{{ }^{1} \mathrm{H}\right\} \mathrm{NMR}(\mathrm{THF}) \delta$ : 34.0 (s), -27.7 (s), 17.7 (s), 119.69 (s), 156.29 (s), 163.71 (s), 200.84 (s).

\section{1:1 Reaction of ${ }^{\mathrm{iPr}} \mathrm{NHC}^{\mathrm{Me}}$ and $\mathrm{PBr}_{3}$}

To a solution of ${ }^{\text {Pr NHC }}{ }^{\mathrm{Me}}(50.0 \mathrm{mg}, 0.277 \mathrm{mmol})$ was added $\mathrm{PBr}_{3}(75.0 \mathrm{mg}, 0.277 \mathrm{mmol})$ in THF (1 mL). Upon addition, a yellow precipitate formed immediately. ${ }^{31} \mathrm{P}\left\{{ }^{1} \mathrm{H}\right\} \mathrm{NMR}(\mathrm{THF}) \delta$ : 54.1 (s), -26.2 (s), 20.7 (s), 140.6 (d), 218.2 (br, $\left.\mathrm{PBr}_{3}\right)$.

\section{1:1 Reaction of ${ }^{\text {iPr }} \mathrm{NHC}^{\mathrm{Me}}$ and $\mathrm{PI}_{3}$}

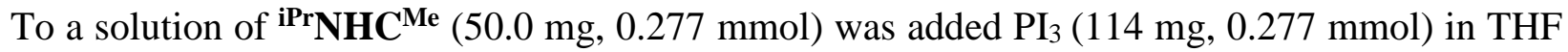
$(1 \mathrm{~mL})$. Upon addition, a yellow precipitate formed immediately. ${ }^{31} \mathrm{P}\left\{{ }^{1} \mathrm{H}\right\} \mathrm{NMR}(\mathrm{THF}) \delta:-21.0$ (s), $100.1\left(\mathrm{~s}, \mathrm{P}_{2} \mathrm{I}_{4}\right), 172.6\left(\mathrm{~s}, \mathrm{PI}_{3}\right)$. 
Table S20 Summary of ${ }^{31} \mathrm{P}$ NMR chemical shifts for 1:1 reactions between carbenes and $\mathrm{PX}_{3}$ (values given are in ppm)

\begin{tabular}{cccc}
\hline Starting Reagents & ${ }^{\text {MeNHC }}{ }^{\text {Me }}$ & EtNHC $^{\mathbf{M e}}$ & ${ }^{\text {iPr}} \mathbf{N H C}^{\mathbf{M e}}$ \\
\hline $\mathrm{PCl}_{3}$ & -115.2 & -99.1 & -34.0 \\
& 218.8 & -30.7 & -27.7 \\
& & 17.0 & 17.7 \\
& & 212.7 & 119.7 \\
& & & 156.3 \\
& & & 163.7 \\
PBr3 & -98.0 & -96.3 & -54.1 \\
& -30.2 & -30.0 & -26.2 \\
& 230.1 & 17.6 & 20.7 \\
& & 225.9 & 140.6 \\
& & & 218.2 \\
\hline PI3 & 102.8 & -80.3 & -21.0 \\
& 174.2 & -27.9 & 100.1 \\
& & 8.1 & 172.6 \\
& & 99.4 & \\
\hline
\end{tabular}

\section{2:1 Reaction of ${ }^{\mathrm{Me}} \mathrm{NHC}^{\mathrm{Me}}$ and $\mathrm{PCl}_{3}$}

To a solution of ${ }^{\mathrm{Me}} \mathrm{NHC}{ }^{\mathrm{Me}}(50.0 \mathrm{mg}, 0.403 \mathrm{mmol})$ was added $\mathrm{PCl}_{3}(28.0 \mathrm{mg}, 0.201 \mathrm{mmol})$ in THF (1 mL). Upon addition, a yellow precipitate formed immediately. ${ }^{31} \mathrm{P}\left\{{ }^{1} \mathrm{H}\right\} \mathrm{NMR}(\mathrm{THF}) \delta$ : $115.2(\mathrm{~s}), 218.1\left(\mathrm{~s}, \mathrm{PCl}_{3}\right)$.

\section{2:1 Reaction of ${ }^{\mathrm{Me}} \mathrm{NHC}{ }^{\mathrm{Me}}$ and PBr3}

To a solution of ${ }^{\mathrm{Me}} \mathrm{NHC}{ }^{\mathrm{Me}}(50.0 \mathrm{mg}, 0.403 \mathrm{mmol})$ was added $\mathrm{PBr}_{3}(54.0 \mathrm{mg}, 0.201 \mathrm{mmol})$ in THF (1 mL). Upon addition, a yellow/orange precipitate formed immediately. ${ }^{31} \mathrm{P}\left\{{ }^{1} \mathrm{H}\right\} \mathrm{NMR}$ (THF) $\delta:-115.1(\mathrm{~s}),-96.6(\mathrm{~s}),-31.3(\mathrm{~s}), 50.27(\mathrm{~s}), 148.08(\mathrm{~s}), 231.49\left(\mathrm{~s}, \mathrm{PBr}_{3}\right)$.

\section{2:1 Reaction of ${ }^{\mathrm{Me}} \mathrm{NHC}^{\mathrm{Me}}$ and $\mathrm{PI}_{3}$}


To a solution of ${ }^{\mathrm{Me}} \mathrm{NHC}^{\mathrm{Me}}(50.0 \mathrm{mg}, 0.403 \mathrm{mmol})$ was added $\mathrm{PI}_{3}(83.0 \mathrm{mg}, 0.201 \mathrm{mmol})$ in THF $(1 \mathrm{~mL})$. Upon addition, a yellow/orange precipitate formed immediately. ${ }^{31} \mathrm{P}\left\{{ }^{1} \mathrm{H}\right\}$ NMR (THF) $\delta: 102.8\left(\mathrm{~s}, \mathrm{P}_{2} \mathrm{I}_{4}\right), 174.2\left(\mathrm{~s}, \mathrm{PI}_{3}\right)$.

\section{2:1 Reaction of ${ }^{\mathrm{Et}} \mathrm{NHC}^{\mathrm{Me}}$ and $\mathrm{PCl}_{3}$}

To a solution of ${ }^{\mathbf{E t}} \mathrm{NHC}^{\mathrm{Me}}(50.0 \mathrm{mg}, 0.328 \mathrm{mmol})$ was added $\mathrm{PCl}_{3}(22.0 \mathrm{mg}, 0.164 \mathrm{mmol})$ in

THF $(1 \mathrm{~mL})$. Upon addition, yellow and dark orange precipitate formed immediately. ${ }^{31} \mathrm{P}\left\{{ }^{1} \mathrm{H}\right\}$ NMR (THF) $\delta:-95.1(\mathrm{~s}),-31.0(\mathrm{~s}), 15.7(\mathrm{~s}), 165.7(\mathrm{~s}), 214.00\left(\mathrm{~s}, \mathrm{PCl}_{3}\right)$.

\section{2:1 Reaction of ${ }^{\mathrm{Et}} \mathrm{NHC}^{\mathrm{Me}}$ and $\mathrm{PBr}_{3}$}

To a solution of ${ }^{\mathrm{Et}} \mathrm{NHC}^{\mathrm{Me}}(50.0 \mathrm{mg}, 0.328 \mathrm{mmol})$ was added $\mathrm{PBr}_{3}(44.0 \mathrm{mg}, 0.164 \mathrm{mmol})$ in THF (1 mL). Upon addition, a yellow precipitate formed immediately. ${ }^{31} \mathrm{P}\left\{{ }^{1} \mathrm{H}\right\} \mathrm{NMR}$ (THF) $\delta$ : $96.3(\mathrm{~s}),-30.2(\mathrm{~s}), 15.9(\mathrm{~s}), 142.1(\mathrm{~d}), 146.3$ (s) $225.9\left(\mathrm{~s}, \mathrm{PBr}_{3}\right)$.

\section{2:1 Reaction of ${ }^{\mathrm{Et}} \mathrm{NHC}^{\mathrm{Me}}$ and $\mathrm{PI}_{3}$}

To a solution of ${ }^{\text {Et }}$ HHC $^{\mathrm{Me}}(50.0 \mathrm{mg}, 0.328 \mathrm{mmol})$ was added $\mathrm{PI}_{3}(68.0 \mathrm{mg}, 0.164 \mathrm{mmol})$ in THF $(1 \mathrm{~mL})$. Upon addition, a dark orange precipitate formed immediately. ${ }^{31} \mathrm{P}\left\{{ }^{1} \mathrm{H}\right\}$ NMR (THF) $\delta$ : $95.0(\mathrm{~s}),-28.0(\mathrm{~s})$.

\section{2:1 Reaction of ${ }^{\mathrm{iPr}} \mathrm{NHC}^{\mathrm{Me}}$ and $\mathrm{PCl}_{3}$}

To a solution of ${ }^{\text {iPr }} \mathbf{N H C}{ }^{\mathrm{Me}}(50.0 \mathrm{mg}, 0.277 \mathrm{mmol})$ was added $\mathrm{PCl}_{3}(19.0 \mathrm{mg}, 0.139 \mathrm{mmol})$ in THF (1 mL). Upon addition, a yellow precipitate formed immediately. ${ }^{31} \mathrm{P}\left\{{ }^{1} \mathrm{H}\right\} \mathrm{NMR}$ (THF) $\delta$ : $122.6(\mathrm{br}),-27.2(\mathrm{~s}), 17.8(\mathrm{~s}), 204.02(\mathrm{~s})$

\section{2:1 Reaction of ${ }^{\text {iPr }} \mathrm{NHC}^{\mathrm{Me}}$ and $\mathrm{PBr}_{3}$}


To a solution of ${ }^{\mathrm{iPr}} \mathbf{N H C}{ }^{\mathrm{Me}}(50.0 \mathrm{mg}, 0.277 \mathrm{mmol})$ was added $\mathrm{PBr}_{3}(38.0 \mathrm{mg}, 0.139 \mathrm{mmol})$ in THF (1 mL). Upon addition, a yellow precipitate formed immediately. ${ }^{31} \mathrm{P}\left\{{ }^{1} \mathrm{H}\right\} \mathrm{NMR}$ (THF) $\delta$ : 122.9 (br), -18.7 (s), $223.2\left(\mathrm{~s}, \mathrm{PBr}_{3}\right)$.

\section{2:1 Reaction of ${ }^{\text {iPr }} \mathrm{NHC}^{\mathrm{Me}}$ and $\mathrm{PI}_{3}$}

To a solution of ${ }^{\text {iPr }} \mathbf{N H C}^{\mathrm{Me}}(50.0 \mathrm{mg}, 0.277 \mathrm{mmol})$ was added $\mathrm{PI}_{3}(57.0 \mathrm{mg}, 0.139 \mathrm{mmol})$ in THF $(1 \mathrm{~mL})$. Upon addition, a dark orange precipitate formed immediately. ${ }^{31} \mathrm{P}\left\{{ }^{1} \mathrm{H}\right\}$ NMR (THF) $\delta$ : $121.3(\mathrm{br}),-43.6(\mathrm{~s}),-21.3(\mathrm{~s})$.

Table S21 Summary of ${ }^{31} \mathrm{P}$ NMR chemical shifts for 2:1 reactions between carbenes and $\mathrm{PX}_{3}$ (values given are in $\mathrm{ppm}$ )

\begin{tabular}{|c|c|c|c|}
\hline Starting Reagents & ${ }^{M e} \mathbf{N H C}^{\mathrm{Me}}$ & ${ }^{\text {Et }} \mathbf{N H C}^{\mathrm{Me}}$ & ${ }^{\mathrm{iPr}} \mathrm{NHC}^{\mathrm{Me}}$ \\
\hline $\mathrm{PCl}_{3}$ & $\begin{array}{c}-115.2 \\
218.1\end{array}$ & $\begin{array}{c}-95.1 \\
-31.0 \\
15.7 \\
165.7 \\
212.7\end{array}$ & $\begin{array}{c}-122.6 \\
-27.2 \\
17.8 \\
204.0\end{array}$ \\
\hline $\mathrm{PBr}_{3}$ & $\begin{array}{c}-115.1 \\
-99.7 \\
-31.3 \\
50.3 \\
148.1 \\
231.5\end{array}$ & $\begin{array}{c}-96.3 \\
-30.2 \\
15.9 \\
142.1 \\
146.3 \\
225.9\end{array}$ & $\begin{array}{c}-122.9 \\
18.7 \\
223.2\end{array}$ \\
\hline $\mathbf{P I}_{3}$ & $\begin{array}{l}102.8 \\
174.2\end{array}$ & $\begin{array}{l}-95.0 \\
-28.0\end{array}$ & $\begin{array}{c}-121.3 \\
-43.7 \\
-21.3\end{array}$ \\
\hline
\end{tabular}

\section{3:1 Reaction of ${ }^{\mathrm{Me}} \mathrm{NHC}^{\mathrm{Me}}$ and $\mathrm{PCl}_{3}$}

To a solution of ${ }^{\mathrm{Me}} \mathrm{NHC}^{\mathrm{Me}}(50.0 \mathrm{mg}, 0.403 \mathrm{mmol})$ was added $\mathrm{PCl}_{3}(28.0 \mathrm{mg}, 0.134 \mathrm{mmol})$ in THF (1 mL). Upon addition, a yellow precipitate formed immediately. ${ }^{31} \mathrm{P}\left\{{ }^{1} \mathrm{H}\right\}$ NMR (THF) $\delta$ : $115.3(\mathrm{~s}), 218.0\left(\mathrm{~s}, \mathrm{PCl}_{3}\right)$. 


\section{3:1 Reaction of ${ }^{\mathrm{Me}} \mathrm{NHC} \mathrm{Ce}^{\mathrm{Me}}$ and $\mathrm{PBr}_{3}$}

To a solution of ${ }^{\mathrm{Me}} \mathrm{NHC}{ }^{\mathrm{Me}}(50.0 \mathrm{mg}, 0.403 \mathrm{mmol})$ was added $\mathrm{PBr}_{3}(36.3 \mathrm{mg}, 0.134 \mathrm{mmol})$ in THF (1 mL). Upon addition, a yellow/orange precipitate formed immediately. ${ }^{31} \mathrm{P}\left\{{ }^{1} \mathrm{H}\right\} \mathrm{NMR}$ (THF) $\delta$ : -115.1 (s), -96.6 (s), -31.3 (s), $50.27(\mathrm{~s}), 148.1(\mathrm{~s}), 231.5\left(\mathrm{~s}, \mathrm{PBr}_{3}\right)$.

\section{3:1 Reaction of ${ }^{\mathrm{Me}} \mathrm{NHC}^{\mathrm{Me}}$ and $\mathrm{PI}_{3}$}

To a solution of ${ }^{\mathrm{Me}} \mathrm{NHC}{ }^{\mathrm{Me}}(50.0 \mathrm{mg}, 0.403 \mathrm{mmol})$ was added $\mathrm{PI}_{3}(55.0 \mathrm{mg}, 0.134 \mathrm{mmol})$ in THF (1 mL). Upon addition, a yellow/orange precipitate formed immediately. ${ }^{31} \mathrm{P}\left\{{ }^{1} \mathrm{H}\right\} \mathrm{NMR}$ (THF) $\delta$ : $102.7\left(\mathrm{~s}, \mathrm{P}_{2} \mathrm{I}_{4}\right), 174.2\left(\mathrm{~s}, \mathrm{PI}_{3}\right)$.

\section{3:1 Reaction of ${ }^{\mathrm{Et}} \mathrm{NHC}^{\mathrm{Me}}$ and $\mathrm{PCl}_{3}$}

To a solution of ${ }^{\mathbf{E t}} \mathbf{N H C}{ }^{\mathrm{Me}}(50.0 \mathrm{mg}, 0.328 \mathrm{mmol})$ was added $\mathrm{PCl}_{3}(15.0 \mathrm{mg}, 0.110 \mathrm{mmol})$ in THF (1 mL). Upon addition, yellow and dark orange precipitate formed immediately. ${ }^{31} \mathrm{P}\left\{{ }^{1} \mathrm{H}\right\}$ NMR (THF) $\delta:-125.2(\mathrm{~s}),-30.8(\mathrm{~s}), 17.1(\mathrm{~s})$.

\section{3:1 Reaction of ${ }^{\mathrm{Et}} \mathrm{NHC}{ }^{\mathrm{Me}}$ and $\mathrm{PBr} 3$}

To a solution of ${ }^{\mathbf{E t}} \mathbf{N H C}{ }^{\mathbf{M e}}(50.0 \mathrm{mg}, 0.328 \mathrm{mmol})$ was added $\mathrm{PBr}_{3}(30.0 \mathrm{mg}, 0.110 \mathrm{mmol})$ in

THF (1 mL). Upon addition, a dark yellow and orange precipitate formed immediately. ${ }^{31} \mathrm{P}\left\{{ }^{1} \mathrm{H}\right\}$ NMR (THF) $\delta:-124.7(\mathrm{~s})$.

\section{3:1 Reaction of ${ }^{\mathrm{Et}} \mathrm{NHC}^{\mathrm{Me}}$ and $\mathrm{PI}_{3}$}

To a solution of ${ }^{\mathbf{E t}} \mathbf{N H C}{ }^{\mathrm{Me}}(50.0 \mathrm{mg}, 0.328 \mathrm{mmol})$ was added $\mathrm{PI}_{3}(45.0 \mathrm{mg}, 0.110 \mathrm{mmol})$ in $\mathrm{THF}$ $(1 \mathrm{~mL})$. Upon addition, a yellow precipitate formed immediately. ${ }^{31} \mathrm{P}\left\{{ }^{1} \mathrm{H}\right\} \mathrm{NMR}(\mathrm{THF}) \delta:-126.1$ (s). 


\section{3:1 Reaction of ${ }^{\text {Pr }} \mathrm{NHC}^{\mathrm{Me}}$ and $\mathrm{PCl}_{3}$}

To a solution of ${ }^{\text {iPr }} \mathbf{N H C}^{\mathrm{Me}}(50.0 \mathrm{mg}, 0.277 \mathrm{mmol})$ was added $\mathrm{PCl}_{3}(13.0 \mathrm{mg}, 0.092 \mathrm{mmol})$ in THF (1 mL). Upon addition, a yellow/orange precipitate formed immediately. ${ }^{31} \mathrm{P}\left\{{ }^{1} \mathrm{H}\right\}$ NMR (THF) $\delta:-122.6(\mathrm{br}),-27.1(\mathrm{~s}), 17.5(\mathrm{~s}), 204.0(\mathrm{~s})$.

\section{3:1 Reaction of ${ }^{\text {iPr }} \mathrm{NHC}^{\mathrm{Me}}$ and $\mathrm{PBr}_{3}$}

To a solution of ${ }^{\text {iPr }} \mathbf{N H C}^{\text {Me }}(50.0 \mathrm{mg}, 0.277 \mathrm{mmol})$ was added $\mathrm{PBr}_{3}(25.0 \mathrm{mg}, 0.092 \mathrm{mmol})$ in THF (1 mL). Upon addition, a yellow precipitate formed immediately. ${ }^{31} \mathrm{P}\left\{{ }^{1} \mathrm{H}\right\}$ NMR (THF) $\delta$ : $123.0(\mathrm{br}),-54.1(\mathrm{~s}),-24.6(\mathrm{~s}), 83.8(\mathrm{~s})$.

\section{3:1 Reaction of ${ }^{\text {iPr }} \mathrm{NHC}^{\mathrm{Me}}$ and $\mathrm{PI}_{3}$}

To a solution of ${ }^{\text {iPr }} \mathrm{NHC}^{\mathrm{Me}}(50.0 \mathrm{mg}, 0.277 \mathrm{mmol})$ was added $\mathrm{PI}_{3}(38.0 \mathrm{mg}, 0.092 \mathrm{mmol})$ in THF $(1 \mathrm{~mL})$. Upon addition, a dark orange precipitate formed immediately. ${ }^{31} \mathrm{P}\left\{{ }^{1} \mathrm{H}\right\}$ NMR (THF) $\delta$ : $122.0(\mathrm{br}),-7.5(\mathrm{~s}), 1.8(\mathrm{~s})$.

Table S22 Summary of ${ }^{31} \mathrm{P}$ NMR chemical shifts for 3:1 reactions between carbenes and $\mathrm{PX}_{3}$ (values given are in $\mathrm{ppm}$ )

\begin{tabular}{|c|c|c|c|}
\hline Starting Reagents & ${ }^{M e} N_{C C}{ }^{\text {Me }}$ & Et NHC $^{\mathrm{Me}}$ & ${ }^{\text {Prr NHC }^{\mathrm{Me}}}$ \\
\hline $\mathbf{P C l}_{3}$ & $\begin{array}{c}-115.3 \\
218.0\end{array}$ & $\begin{array}{c}-125.2 \\
-30.8 \\
17.1\end{array}$ & $\begin{array}{c}-122.6 \\
-27.1 \\
17.5 \\
204.0 \\
\end{array}$ \\
\hline PBr3 & $\begin{array}{c}-115.1 \\
-96.6 \\
-31.3 \\
50.3 \\
148.1 \\
231.5\end{array}$ & -124.7 & $\begin{array}{c}-123.0 \\
-54.1 \\
-24.6 \\
83.8\end{array}$ \\
\hline $\mathbf{P I}_{3}$ & $\begin{array}{l}102.7 \\
174.2\end{array}$ & -126.1 & $\begin{array}{c}-122.0 \\
-7.5 \\
1.8 \\
\end{array}$ \\
\hline
\end{tabular}




\section{5:1 Reaction of ${ }^{\mathrm{Me}} \mathrm{NHC}{ }^{\mathrm{Me}}$ and $\mathrm{PCl}_{3}$}

To a solution of ${ }^{\mathrm{Me}} \mathrm{NHC}{ }^{\mathrm{Me}}(50.0 \mathrm{mg}, 0.403 \mathrm{mmol})$ was added $\mathrm{PCl}_{3}(11.1 \mathrm{mg}, 0.081 \mathrm{mmol})$ in

THF (1 mL). Upon addition, a yellow precipitate formed immediately. ${ }^{31} \mathrm{P}\left\{{ }^{1} \mathrm{H}\right\} \mathrm{NMR}(\mathrm{THF}) \delta$ : $115.5(\mathrm{~s}), 217.8\left(\mathrm{~s}, \mathrm{PCl}_{3}\right)$.

\section{5:1 Reaction of ${ }^{\mathrm{Me}} \mathrm{NHC}^{\mathrm{Me}}$ and $\mathrm{PBr}_{3}$}

To a solution of ${ }^{\mathrm{Me}} \mathrm{NHC}{ }^{\mathrm{Me}}(50.0 \mathrm{mg}, 0.403 \mathrm{mmol})$ was added $\mathrm{PBr}_{3}(22.5 \mathrm{mg}, 0.081 \mathrm{mmol})$ in THF (1 mL). Upon addition, a dark orange precipitate formed immediately. ${ }^{31} \mathrm{P}\left\{{ }^{1} \mathrm{H}\right\} \mathrm{NMR}$ (THF) $\delta:-113.4(\mathrm{~s}), 230.8\left(\mathrm{~s}, \mathrm{PBr}_{3}\right)$.

\section{5:1 Reaction of ${ }^{\mathrm{Me}} \mathrm{NHC}^{\mathrm{Me}}$ and $\mathrm{PI}_{3}$}

To a solution of ${ }^{\mathrm{Me}} \mathrm{NHC}{ }^{\mathrm{Me}}(50.0 \mathrm{mg}, 0.403 \mathrm{mmol})$ was added $\mathrm{PI}_{3}(33.0 \mathrm{mg}, 0.081 \mathrm{mmol})$ in THF (1 mL). Upon addition, a yellow/orange precipitate formed immediately. ${ }^{31} \mathrm{P}\left\{{ }^{1} \mathrm{H}\right\} \mathrm{NMR}$ (THF) $\delta$ : $104.3\left(\mathrm{~s}, \mathrm{P}_{2} \mathrm{I}_{4}\right), 121.2(\mathrm{~s}), 175.7\left(\mathrm{~s}, \mathrm{PI}_{3}\right)$.

\section{5:1 Reaction of ${ }^{\mathrm{Et}} \mathrm{NHC}{ }^{\mathrm{Me}}$ and $\mathrm{PCl}_{3}$}

To a solution of ${ }^{\mathbf{E t}} \mathbf{N H C}{ }^{\mathrm{Me}}(50.0 \mathrm{mg}, 0.328 \mathrm{mmol})$ was added $\mathrm{PCl}_{3}(9.00 \mathrm{mg}, 0.066 \mathrm{mmol})$ in THF (1 mL). Upon addition, yellow and dark orange precipitate formed immediately. ${ }^{31} \mathrm{P}\left\{{ }^{1} \mathrm{H}\right\}$ NMR (THF) $\delta:-124.1(\mathrm{~s})$.

\section{5:1 Reaction of ${ }^{\mathrm{Et}} \mathrm{NHC}{ }^{\mathrm{Me}}$ and $\mathrm{PBr}_{3}$}

To a solution of ${ }^{\mathbf{E t}} \mathbf{N H C}{ }^{\mathrm{Me}}(50.0 \mathrm{mg}, 0.328 \mathrm{mmol})$ was added $\mathrm{PBr}_{3}(18.0 \mathrm{mg}, 0.066) \mathrm{THF}(1 \mathrm{~mL})$. Upon addition, a dark orange precipitate formed immediately. ${ }^{31} \mathrm{P}\left\{{ }^{1} \mathrm{H}\right\} \mathrm{NMR}$ (THF) $\delta:-124.8$ (s), $119.4(\mathrm{~s})$. 


\section{5:1 Reaction of ${ }^{\mathrm{Et}} \mathrm{NHC}{ }^{\mathrm{Me}}$ and $\mathrm{PI}_{3}$}

To a solution of EtNHCMe $(50.0 \mathrm{mg}, 0.328 \mathrm{mmol})$ was added $\mathrm{PI}_{3}(27.0 \mathrm{mg}, 0.066 \mathrm{mmol})$ in THF $(1 \mathrm{~mL})$. Upon addition, a dark orange precipitate formed immediately. ${ }^{31} \mathrm{P}\left\{{ }^{1} \mathrm{H}\right\} \mathrm{NMR}(\mathrm{THF}) \delta$ : $125.3(\mathrm{~s}), 119.7$ (s).

\section{5:1 Reaction of ${ }^{\mathrm{iPr}} \mathrm{NHC}^{\mathrm{Me}}$ and $\mathrm{PCl}_{3}$}

To a solution of ${ }^{\text {Pr}} \mathbf{N H C}{ }^{\mathrm{Me}}(50.0 \mathrm{mg}, 0.277 \mathrm{mmol})$ was added $\mathrm{PCl}_{3}(7.62 \mathrm{mg}, 0.055 \mathrm{mmol})$ in THF (1 mL). Upon addition, a dark orange precipitate formed immediately. ${ }^{31} \mathrm{P}\left\{{ }^{1} \mathrm{H}\right\} \mathrm{NMR}$ (THF) $\delta:-122.3(\mathrm{br})$.

\section{5:1 Reaction of ${ }^{\text {iPr }} \mathrm{NHC}^{\mathrm{Me}}$ and $\mathrm{PBr}_{3}$}

To a solution of ${ }^{\text {Pr NHC }}{ }^{\mathrm{Me}}(50.0 \mathrm{mg}, 0.277 \mathrm{mmol})$ was added $\mathrm{PBr}_{3}(15.0 \mathrm{mg}, 0.055 \mathrm{mmol})$ in THF (1 mL). Upon addition, a dark orange precipitate formed immediately. ${ }^{31} \mathrm{P}\left\{{ }^{1} \mathrm{H}\right\} \mathrm{NMR}$ (THF) $\delta:-122.6(\mathrm{br}),-54.13(\mathrm{~s}),-25.69(\mathrm{~s})$.

\section{5:1 Reaction of ${ }^{\mathrm{iPr}} \mathrm{NHC}^{\mathrm{Me}}$ and PI3}

To a solution of ${ }^{\text {Prr NHC }}{ }^{\mathrm{Me}}(50.0 \mathrm{mg}, 0.277 \mathrm{mmol})$ was added $\mathrm{PI}_{3}(23.0 \mathrm{mg}, 0.055 \mathrm{mmol})$ in $\mathrm{THF}$ $(1 \mathrm{~mL})$. Upon addition, a dark orange precipitate formed immediately. ${ }^{31} \mathrm{P}\left\{{ }^{1} \mathrm{H}\right\} \mathrm{NMR}$ (THF) $\delta$ : 122.2 (br), $-43.6(\mathrm{~s})$.

Table S23 Summary of ${ }^{31} \mathrm{P}$ NMR chemical shifts for 5:1 reactions between carbenes and $\mathrm{PX}_{3}$ (values given are in $\mathrm{ppm}$ )

\begin{tabular}{cccc}
\hline Starting Reagents & MeNHC $^{\text {Me }}$ & EtNHC $^{\text {Me }}$ & iPr NHC $^{\text {Me }}$ \\
\hline PCl3 $_{3}$ & -115.5 & -124.1 & -122.3 \\
& 217.8 & & \\
\hline PBr3 & -113.4 & -124.8 & -122.6 \\
& 230.8 & 119.4 & -54.1 \\
& & & -25.7 \\
\hline
\end{tabular}




\begin{tabular}{cccc}
\hline PI3 & 104.3 & -125.3 & -122.0 \\
& 121.2 & 120.1 & -43.6 \\
& 174.2 & & \\
\hline
\end{tabular}




\section{References}

$1 \quad$ R. Allmann, Angew. Chem., 1965, 77, 134-135.

2 K. Schwedtmann, M. H. Holthausen, K.-O. Feldmann and J. J. Weigand, Angew. Chem. Int. Ed., 2013, 52, 14204-8.

3 J. F. Binder, A. M. Corrente and C. L. B. Macdonald, Dalton Trans., 2016, 45, 2138-2147.

4 I. Kawada and R. Allmann, Angew. Chem. Int. Ed., 1968, 7, 69-69.

5 A. Schmidpeter, R. O. Day, A. Willhalm, J. M. Holmes and R. R. Holmes, Angew. Chem. Int. Ed., 1985, 24, 764-765.

B. D. Ellis, C. A. Dyker, A. Decken and C. L. B. Macdonald, Chem. Commun., 2005, 1965-1967.

A. Schulz, A. Villinger and A. Hinz, Chem. Commun., 2016.

M. Cicač-Hudi, J. Bender, S. H. Schlindwein, M. Bispinghoff, M. Nieger, H. Grützmacher and D. Gudat, Eur. J. Inorg. Chem., 2016, 2016, 649-658.

9 T. Stey, M. Pfeiffer, J. Henn, S. K. Pandey and D. Stalke, Chem. Eur. J., 2007, 13, 3636-3642.

10 A. Steiner and D. Stalke, Organometallics, 1995, 14, 2422-2429.

11 T. Stey, J. Henn and D. Stalke, Chem. Commun., 2007, 413-415.

12 F. Lindenberg, J. Sieler and E. Hey-hawkins, Phosphorus Sulfur Silicon Relat. Elem., 2006, 108, 279-283.

13 B. Hoge and W. Wiebe, Angew. Chem. Int. Ed., 2008, 47, 8116-9.

14 J. F. Binder, A. Swidan, M. Tang, J. H. Nguyen and C. L. B. Macdonald, Chem. Commun., 2015, 51, 7741-7744.

15 M. J. Frisch, G. W. Trucks, H. B. Schlegel, G. E. Scuseria, M. A. Robb, J. R. Cheeseman, G. Scalmani, V. Barone, B. Mennucci, G. A. Petersson, H. Nakatsuji, M. Caricato, X. Li, H. P. Hratchian, A. F. Izmaylov, J. Bloino, G. Zheng, J. L. Sonnenberg, M. Hada, M. Ehara, K. Toyota, R. Fukuda, J. Hasegawa, M. Ishida, T. Nakajima, Y. Honda, O. Kitao, H. Nakai, T. Vreven, J. A. Montgomery, J., J. E. Peralta, F. Ogliaro, M. Bearpark, J. J. Heyd, E. Brothers, K. N. Kudin, V. N. Staroverov, R. Kobayashi, J. Normand, K. Raghavachari, A. Rendell, J. C. Burant, S. S. Iyengar, J. Tomasi, M. Cossi, N. Rega, N. J. Millam, M. Klene, J. E. Knox, J. B. Cross, V. Bakken, C. Adamo, J. Jaramillo, R. Gomperts, R. E. Stratmann, O. Yazyev, A. J. Austin, R. Cammi, C. Pomelli, J. W. Ochterski, R. L. Martin, K. Morokuma, V. G. Zakrzewski, G. A. Voth, P. Salvador, J. J. Dannenberg, S. Dapprich, A. D. Daniels, Ö. Farkas, J. B. Foresman, J. V. Ortiz, J. Cioslowski and D. J. Fox, 2009, Gaussian09, Revision D.01, Gaussian Inc. Wallingford, CT.

J. Perdew, K. Burke and M. Ernzerhof, Phys. Rev. Lett., 1996, 77, 3865-3868.

J. P. Perdew, K. Burke and M. Ernzerhof, Phys. Rev. Lett., 1997, 1396.

C. Adamo and V. Barone, J. Chem. Phys., 1999, 110, 6158.

19 A. Schafer, H. Horn and R. Ahlrichs, J. Chem. Phys., 1992, 97, 2571-2577. 
20 A. Schafer, C. Huber and R. Ahlrichs, J. Chem. Phys., 1994, 100, 5829.

21 Gaussview 3.0, Gaussian Inc., Pittsburgh, PA, 2003.

22 A. E. Reed, L. A. Curtiss and F. Weinhold, Chem. Rev., 1988, 88, 899-926.

23 NBO 3.0, E. D. Glendening, A. E. Reed, J. E. Carpenter and F. Weinhold, .

24 F. B. Konig, F.B., J. Schonbohm, J. and D. Bayles, D., J. Comput. Chem., 2001, 22, 545-559. 